\title{
Proton pump inhibitors enhance intestinal permeability via dysbiosis of gut microbiota under stressed conditions in mice
}

Shingo Takashima, Fumio Tanaka, Yunosuke Kawaguchi, Yuki Usui, Kosuke Fujimoto, Yuji Nadatani, Koji Otani, Shuhei Hosomi, Yasuaki Nagami, Noriko Kamata, Koichi Taira, Tetsuya Tanigawa, Toshio Watanabe, Seiya Imoto, Satoshi Uematsu, Yasuhiro Fujiwara

\begin{tabular}{|c|l|}
\hline Citation & Neurogastroenterology and Motility. 32(7); e13841 \\
\hline Issue Date & $2020-06-25$ \\
\hline Version of Record & $2020-04-21$ \\
\hline Type & Journal Article \\
\hline Textversion & Author \\
\hline $\begin{array}{c}\text { Supporting } \\
\text { Information }\end{array}$ & Supporting Information is available at $\underline{\text { https://doi.org/10.1111/nmo.13841. }}$ \\
\hline Rights & $\begin{array}{l}\text { This is the peer reviewed version of the following article: } \\
\text { Neurogastroenterology and Motility. Vol.32, Issu.7, e13841, which has been } \\
\text { published in final form at https://doi.org/10.1111/nmo.13841. This article } \\
\text { may be used for non-commercial purposes in accordance with Wiley Terms } \\
\text { and Conditions for Use of Self-Archived Versions. }\end{array}$ \\
\hline DOI & \begin{tabular}{l}
$10.1111 / n m o .13841$ \\
\hline
\end{tabular}
\end{tabular}

\section{Self-Archiving by Author(s)}

Placed on: Osaka City University Repository

TAKASHIMA, S., et al. (2020). Proton pump inhibitors enhance intestinal permeability via dysbiosis of gut microbiota under stressed conditions in mice. Neurogastroenterology \& Motility. 32. e13841. Doi: $10.1111 / \mathrm{nmo.13841}$ 
Proton pump inhibitors enhance intestinal permeability via dysbiosis of gut microbiota under stressed conditions in mice

Shingo Takashima ${ }^{1}$, Fumio Tanaka ${ }^{1}$, Yunoske Kawaguchi ${ }^{2}$, Yuki Usui ${ }^{3}$, Kosuke Fujimoto $^{2,4}$, Yuji Nadatani ${ }^{1}$, Koji Otani ${ }^{1}$, Shuhei Hosomi ${ }^{1}$, Yasuaki Nagami ${ }^{1}$, Noriko Kamata $^{1}$, Koichi Taira ${ }^{1}$, Tetsuya Tanigawa ${ }^{1}$, Toshio Watanabe ${ }^{1}$, Seiya Imoto $^{5}$, Satoshi Uematsu $^{2,4}$, and Yasuhiro Fujiwara ${ }^{1}$

1) Department of Gastroenterology, Osaka City University Graduate School of Medicine, Osaka, Japan

2) Department of Immunology and Genomics, Osaka City University Graduate School of Medicine, Osaka, Japan

3) Division of Systems Immunology, The Institute of Medical Science, The University of Tokyo, Tokyo, Japan

4) Division of Innate Immune Regulation, International Research and Development Center for Mucosal Vaccines, The Institute of Medical Science, The University of Tokyo, Tokyo, Japan 
5) Division of Health Medical Data Science, Health Intelligence Center, The Institute of Medical Science, The University of Tokyo, Tokyo, Japan

Corresponding Author: Fumio Tanaka, MD, PhD

Department of Gastroenterology, Osaka City University Graduate School of Medicine,

Osaka, Japan

1-4-3 Asahimachi, Abeno-ku, Osaka 545-8585, Japan

Phone: +81-6-6645-3811, Fax: +81-6-6645-3813

E-mail: m2079981@med.osaka-cu.ac.jp

Short running title: PPI and intestinal permeability 


\begin{abstract}
Background: Intestinal permeability and psychological stress are considered the key mechanism(s) in functional dyspepsia (FD). Although proton pump inhibitors (PPIs) are commonly used for the treatment of FD, the effect of PPIs on intestinal permeability has not been elucidated. This study investigated the effect of PPI on intestinal permeability under stressed conditions.
\end{abstract}

Methods: C57BL/6J mice were subjected to water avoidance stress (WAS) and administered rabeprazole (40 $\mathrm{mg} \mathrm{kg}^{-1}$ ) or vehicle treatment (VT). We then evaluated intestinal permeability both in vivo and ex vivo using plasma fluorescein isothiocyanatedextran and by assessing the paracellular permeability and transepithelial electrical resistance (TEER) in an Ussing chamber, respectively. Furthermore, we evaluated the effect of PPI-treated fecal microbiota transplant (FMT) on intestinal permeability in vivo. Microbiota profiles of donor feces were assessed by 16S rRNA gene analysis using MiSeq and QIIME2.

Key Results: In the WAS treatment, PPI significantly enhanced intestinal permeability in vivo compared to that in VT. Moreover, PPI significantly increased paracellular permeability, and decreased TEER in the duodenum and jejunum, respectively, compared to those in VT under stressed conditions. Moreover, both vasoactive intestinal peptide 
(VIP) receptor antagonist and ketotifen significantly reversed the effect of PPI on intestinal permeability. Furthermore, PPI-treated FMT significantly increased the intestinal permeability in vivo compared to that in vehicle-treated FMT. PPI treatment altered the gut microbiota composition, indicating that PPI induced dysbiosis.

Conclusions \& Inferences: Under stressed conditions, PPI enhances intestinal permeability via dysbiosis of gut microbiota. VIP and mast cells are also implicated in the underlying mechanisms.

Keywords: Proton pump inhibitors, Permeability, Psychological stress, Fecal microbiota transplantation, Dysbiosis, Mast cells, Vasoactive intestinal peptide 


\section{INTRODUCTION}

The pathophysiology of functional dyspepsia (FD) is characterized by multifactorial disorders of the upper gastrointestinal (GI) tract. Of these, increased intestinal permeability and psychological stress are key factors. ${ }^{1-3}$ In patients with FD, low-grade inflammation was observed in the duodenum and it was associated with increased intestinal permeability. Psychological stress also enhances intestinal permeability in rodents and humans. ${ }^{4}$ Psychological stress induces the release of corticotropin-releasing hormone $(\mathrm{CRH})$, which increases the intestinal permeability by a mast cell-dependent mechanism. ${ }^{5}$ Moreover, vasoactive intestinal peptide (VIP) and its receptor (vasoactive intestinal peptide receptor; VPAC) are involved in stress-induced intestinal barrier dysfunction and have been implicated in the control of paracellular permeability. ${ }^{6}$ VIP is a peptide hormone found in non-cholinergic secretomotor/vasodilator neurons and VPAC is expressed on mast cells. VIP-containing neurons directly innervate the epithelium and regulate ion and fluid secretion and paracellular permeability. ${ }^{7-9}$ Therefore, under stressed conditions, CRH-mast cells-VIP axis is important for enhancement of intestinal permeability.

As a treatment option of FD, proton pump inhibitors (PPIs) are usually used according to the American College of Gastroenterology (ACG) and the Canadian Association of 
Gastroenterology (CAG) guidelines. ${ }^{10}$ In the Japanese guidelines published by the Japanese Society of Gastroenterology, PPIs are also recommended for the first-line treatment of FD. ${ }^{11}$ However, there is a possibility of PPIs causing dysbiosis of gut microbiota due to gastric acid suppression. ${ }^{12,13}$ In a recent study, gut microbiota was considered as one of the factors responsible for enhancement of intestinal permeability. ${ }^{14}$ Therefore, we hypothesized that PPI treatment affects intestinal permeability through alteration of gut microbiota. To date, there are few studies describing the association between PPIs and intestinal permeability, despite intestinal permeability being an important factor in the pathophysiology of FD. In 2008, Mullin et al. reported that esomeprazole enhanced the intestinal permeability of the upper GI tract in healthy volunteers; however, the mechanism was unclear. ${ }^{15}$ Since these participants might have been stressed when they underwent the experiments, the results might have been affected by not only the PPIs but also stress. Whether PPIs affect intestinal permeability has not been fully elucidated, especially under stressed conditions. Keita et al. reported stressinduced ileal bacterial translocation, which was mediated by VIP. ${ }^{6}$ Therefore, stress might enhance the effects of dysbiosis via VIP. The aim of our study was to investigate whether PPI affects the intestinal permeability under stressed conditions in mice, and clarify the role of stress and gut microbiota in the intestinal permeability. 


\section{MATERIALS AND METHODS}

\subsection{Ethics and animals}

Male C57BL/6J mice, aged 6 weeks and weighing approximately $20 \mathrm{~g}$, were purchased from Japan SLC, Inc. (Shizuoka, Japan). All the mice were housed in plastic cages and maintained under a 12-h light and dark cycle with AIN-76A Purified Diet (D10001 from Research Diets Inc.) and water ad libitum. They were randomly assigned to various groups. The experimental protocol was approved by the Animal Care and Use Committees of Osaka City University (No. 17021).

\subsection{Psychological stress and induction of PPI}

During the water avoidance stress (WAS) treatment, each mouse was placed on a circular platform $(4 \mathrm{~cm}$ in diameter $)$ located at the center of a standard plastic cage $(420 \times 300 \times 310$ $\mathrm{mm})$ which was filled with tap water $(20 \mathrm{~mL})$ to $1 \mathrm{~cm}$ below the surface of the platform for $1 \mathrm{~h} \mathrm{day}^{-1}$ and repeated for 8 consecutive days. During the sham stress (SS) treatment, each mouse was placed in a similar cage without water for the same period as previously reported. ${ }^{16}$ Rabeprazole (RPZ, Wako Pure Chemical Industries, Ltd., Osaka, Japan) was diluted to a concentration of $1 \mathrm{mg} \mathrm{mL}^{-1}$ using carboxymethyl cellulose (CMC, Wako Pure Chemical Industries, Ltd., Osaka, Japan, $\left.5 \mathrm{mg} \mathrm{mL} \mathrm{m}^{-1}\right)$. Next, RPZ (40 $\mathrm{mg} \mathrm{kg}^{-1}$ ) was 
administered intraperitoneally just after the stress session for 7 consecutive days. The mice in the vehicle treatment (VT) group were administered the same concentration of the CMC solution. The mice were divided into 4 groups at random: SS+VT, SS+PPI, WAS+VT, and WAS+PPI. The mice of all the 4 groups and their food intake were weighed daily. Change in weight and food intake was calculated and expressed in grams. Food intake was calculated as the average of the daily intake by 5 animals. In another set of experiments, we evaluated the intestinal permeability in vivo using treatments with 40 $\mathrm{mg} \mathrm{mL} \mathrm{mL}^{-1}$ of omeprazole (OPZ, Wako Pure Chemical Industries, Ltd., Osaka, Japan), lansoprazole (LPZ, Wako Pure Chemical Industries, Ltd., Osaka, Japan), and vonoprazan (VPZ, ChemScene, NJ, USA), a potassium-competitive acid blocker (P-CAB).

\subsection{Tissue isolation}

Mice were anesthetized using $2 \%$ isoflurane and sacrificed on day 8 , followed by the isolation of the jejunal and duodenal tissues. For Ussing chamber experiments, the tissues were immediately immersed in ice-cold oxygenated Krebs buffer containing (mM): 115.0 $\mathrm{NaCl}, 1.25 \mathrm{CaCl}_{2}, 1.20 \mathrm{MgCl}_{2}, 2.0 \mathrm{KH}_{2} \mathrm{PO}_{4}, 25.0 \mathrm{NaHCO}_{3}$, and $10.0 \mathrm{C}_{6} \mathrm{H}_{12} \mathrm{O}_{6}(\mathrm{pH}$ 7.35). Tissue segments $(2.4 \times 7.0 \mathrm{~mm})$ were immediately frozen on dry ice and stored at $-80^{\circ} \mathrm{C}$ for protein analysis. For histological evaluation and immunofluorescence staining, the 
tissues were preserved in 4\%-paraformaldehyde phosphate buffer solution (Wako Pure

Chemical Industries, Ltd., Osaka, Japan) and embedded in paraffin. For quantitative reverse transcriptase-polymerase chain reaction (qRT-PCR), the tissues were stored in RNA later solution (Applied Biosystems, Foster, CA, USA) at $-20^{\circ} \mathrm{C}$.

\subsection{Histological evaluation and immunofluorescence for mast cells}

Tissue segments were stained with Mayer's Hematoxylin and Eosin Y solution (H\&E) and scored in a blind manner for histological inflammation and crypt damage using the disease activity index (DAI) as described previously. ${ }^{17,18}$ Immunofluorescence was performed for mast cell tryptase. Mast cell counts were represented as the average number of mast cells in 5 high-power fields of view.

\subsection{Enzyme-linked immunosorbent assay}

Enzyme-linked immunosorbent assay (ELISA) was performed to evaluate the VIP concentrations in the duodenum using an ELISA kit (Phoenix Pharmaceuticals, Karlsruhe, Germany). ELISA was also performed to determine the serum corticosterone concentrations using an ELISA kit (ENZO, Life Sciences, Farmingdale, New York, USA). Serum was collected on day 8 and stored in $0.5 \mathrm{~mL}$ aliquots at $-30^{\circ} \mathrm{C}$ until analyses were 
performed.

\section{6 mRNA and protein expressions of cell-to-cell adhesion molecules}

The levels of mRNA expression of occludin, zonula occludens (ZO)-1, and junctional adhesion molecule-A (JAM-A) in the duodenum and jejunum were measured by qRTPCR. The levels of expression of these mRNAs are indicated as ratios to the mean value of the SS+VT group. In addition, western blot methods were used to quantify the protein expressions of ZO-1 and occludin. The expression level of each protein was normalized to that of $\beta$-actin.

\subsection{Measurement of intestinal permeability in vivo}

Fluorescein isothiocyanate (FITC)-dextran (dx) (molecular weight -4,000 Da, SigmaAldrich, St. Louis, MO, USA) was dissolved in phosphate-buffered saline (PBS) to the concentration of $15 \mathrm{mM}$ in dark conditions. The mice were given $600 \mathrm{mg} \mathrm{kg}^{-1}$ body weight of FITC-dx via the oral route $6 \mathrm{~h}$ after initiating fasting conditions. Blood was collected from the postcaval veins of the mice $4 \mathrm{~h}$ after administration of FITC-dx. The plasma was obtained as supernatant following the centrifugation of the blood samples at $4{ }^{\circ} \mathrm{C}$, $12,000 \times \mathrm{g}$, for $3 \mathrm{~min}$. The FITC-dx concentration of plasma was analyzed with a 
fluorescence spectrophotometer (Varioskan LUX, Thermo Fisher Scientific, Rockford, IL, USA) at an excitation wavelength of $485 \mathrm{~nm}$ and an emission wavelength of $535 \mathrm{~nm}$.

\subsection{Measurement of intestinal permeability ex vivo, Method 1: Transepithelial} electrical resistance

Duodenal (1-4 cm from the pylorus) and jejunal (3-7 cm from the anal side of the Treitz ligament) tissues were opened along the mesenteric border and the tissue segments (5/animal) were mounted in Ussing chambers (Warner Instruments, CT, USA) with an aperture of $16.8 \mathrm{~mm}^{2}$. Next, the transepithelial electrical resistance (TEER) was measured to evaluate the intestinal permeability ex vivo.

\subsection{Measurement of intestinal permeability ex vivo, Method 2: Macromolecular}

\section{transport}

Macromolecular transport was assessed by measuring the flux of FITC-dx from the mucosal to serosal sides of the duodenal and jejunal tissues within the Ussing chamber, which represented the paracellular permeability. A colorimetric endpoint assay was used to quantify the amount of FITC contained in each sample. The concentration of FITC present in each sample was determined using a standard curve. FITC-dx flux was 
expressed as ngFITC/h/mm².

\subsection{Effect of VIP receptor antagonist and mast cell stabilizer on intestinal permeability in WAS +PPI-treated mice}

The effect of VIP receptor antagonist (anti-VPAC) and a mast cell stabilizer, ketotifen fumarate $(\mathrm{KF})$, on the WAS+PPI-induced changes in intestinal permeability was evaluated. Moreover, we evaluated the mast cell count in the duodenums of WAS+PPI+ anti-VPAC mice and the VIP concentrations in the duodenums of WAS+PPI+KF mice. These drugs were administered intraperitoneally following the stress and PPI administration sessions for 7 consecutive days.

\subsection{Effect of microbiota transplantation on intestinal permeability and mast cells}

In order to investigate the role of gut microbiota on the effects of PPI, we performed fecal microbiota transplantation (FMT). The administration of antibiotics and microbial contents was conducted according to a protocol used in previous studies. ${ }^{19,20}$ The mice used in these studies were not housed in groups. The antibiotic cocktail $\left(1 \mathrm{~g} \mathrm{~L}^{-1}\right.$ ampicillin, $500 \mathrm{mg} \mathrm{L}^{-1}$ vancomycin, $1 \mathrm{~g} \mathrm{~L}^{-1}$ neomycin sulfate, and $1 \mathrm{~g} \mathrm{~L}^{-1}$ metronidazole) in drinking water was administered orally to the mice for 28 days ad libitum. ${ }^{21,22}$ This cocktail was 
used to reduce the levels of all detectable intestinal microbiota. Antibiotic feces consisted of the contents of the small intestine that were collected from the antibiotic-treated mice. The small intestinal microbial contents of the donor mice (vehicle-treated mice and PPItreated mice) were harvested and rapidly stirred in $1.5 \mathrm{ml}$ PBS. The small intestinal contents were then orally administered to stressed mice via gastric gavage tube once a day for 7 consecutive days. We ensured that no intestinal bacterial contamination was present during oral administration. The intestinal permeability and mast cell counts were then compared between the mice that received vehicle feces and those that received PPI feces. Vehicle feces consisted of the contents of the small intestine collected from the vehicle-treated donor mice. PPI feces consisted of the contents of the small intestine collected from the PPI-treated donor mice.

\subsection{S rRNA gene analysis}

Bacterial DNA from each sample was extracted as described previously. ${ }^{23}$ The $16 \mathrm{~S}$ rRNA V3-V4 region was amplified by PCR and purified as described previously. ${ }^{24}$ Microbiota profiles were assessed by 16S rRNA gene analysis using MiSeq and QIIME2.

\subsection{Statistics}


All results were expressed as mean \pm standard error. Statistical analyses were performed using the Student's t test or One-way ANOVA followed by the Holm post hoc analysis. All statistical analyses were performed using EZR (Saitama Medical Center, Jichi Medical University), which is a graphical user interface for R (The R Foundation for Statistical Computing, version 2.13.0). ${ }^{25}$ A $P$ value less than 0.05 was considered as significant.

Detailed methods are described in Supplementary Methods. 


\section{RESULTS}

3.1 WAS and PPI caused no changes in body weight, food intake, and microscopic inflammation of the duodenum and jejunum

There was no significant difference among the 4 groups with regard to change in weight and food intake (Figure 1A, B). Photomicrographs of H\&E stained duodenal and jejunal tissue sections are shown in Figure 1C, D. In the VT group, there was no significant difference in microscopic inflammation in the duodenum and jejunum tissues between the WAS and SS groups (Figure 1E, F). In addition, in the WAS group, there was no significant difference in microscopic inflammation in the duodenum and jejunum tissues between the PPI and VT groups.

\subsection{WAS increased the levels of serum corticosterone}

In the VT group, WAS significantly increased the levels of serum corticosterone by $53 \%$ compared to those of the SS group (Figure 2A), which indicated that WAS was properly performed in our experiments. WAS+PPI group also significantly increased the levels of serum corticosterone by $52 \%$ compared to those of the SS+VT group.

\subsection{PPI enhanced the stress-induced increase in VIP levels in the duodenum}


In the VT group, WAS significantly increased the duodenal VIP levels by 55\% compared to those in the SS group (Figure 2B). In the WAS group, PPI significantly increased the duodenal VIP levels by $35 \%$ compared to those in the VT group.

\subsection{PPI enhanced the stress-induced increase in mast cell infiltration in the}

\section{duodenum}

The number of duodenal mast cells in the 4 groups is shown in Figure $2 \mathrm{C}$, D. In the VT group, WAS significantly increased the number of duodenal mast cells by 3.6 -fold compared to the SS group. In the WAS group, PPI significantly enhanced the stressinduced increase in mast cell counts by 1.9-fold compared to that in the VT group.

\subsection{PPI enhanced the stress-induced increase in intestinal permeability in vivo}

In the VT group, WAS significantly increased the plasma FITC-dx concentration by $40 \%$ compared to that in the SS group (Figure 3A), which indicated that stress treatment increased the intestinal permeability in vivo. In the SS group, PPI (RPZ) treatment did not increase the intestinal permeability compared to that in the VT group. In the WAS group, PPI significantly increased the FITC-dx concentration, which indicated that PPI enhanced the stress-induced increase in intestinal permeability in vivo by $30 \%$ compared to that in 
the VT group. Moreover, in the SS group, OPZ and LPZ treatments did not increase the intestinal permeability compared to that in the VT group (Supplementary figure 1A and B). In contrast, in the WAS group, OPZ and LPZ treatments significantly enhanced the stress-induced increase in the intestinal permeability in vivo by $28 \%$ and $18 \%$, respectively, compared to that in the VT group. Taken together, both OPZ and LPZ exerted effects that were similar to those of RPZ, indicating that the observed results may be due to class effects of PPIs.

\subsection{VPZ enhanced the stress-induced increase in intestinal permeability in vivo}

In the VT group, WAS significantly increased the plasma FITC-dx concentration by $40 \%$ compared to that in the SS group (Figure 3B). In the WAS group, VPZ significantly enhanced the stress-induced increase in the intestinal permeability in vivo by $57 \%$ compared to that in the VT group. These results were similar to those obtained from the PPIs.

\subsection{PPI enhanced the stress-induced increase in intestinal permeability ex vivo}

The Ussing chamber experiments showed stable transepithelial potential difference after equilibration in the duodenal and jejunal tissues. In the VT group, WAS significantly 
increased the FITC-dx flux by 8.5 -fold in the duodenum (Figure 3C) and 4.7-fold in the jejunum (Figure 3D), and decreased the TEER by $31 \%$ in the duodenum (Figure 3E) and $38 \%$ in the jejunum (Figure 3F) compared to those in the SS group. In the WAS group, PPI significantly increased the FITC-dx flux by 2.7 -fold in the duodenum and 2.4-fold in the jejunum, and decreased the TEER by $43 \%$ in the duodenum and $29 \%$ in the jejunum compared to those in the VT group. Therefore, PPI enhanced the stress-induced increase in intestinal permeability in both the in vivo and ex vivo models. Furthermore, PPI did not increase intestinal permeability under non-stressed condition in both the in vivo and $e x$ vivo models.

\subsection{PPI decreased mRNA and protein expressions of cell-to-cell adhesion molecules}

\section{in the duodenum}

The mRNA expressions of cell-to-cell adhesion molecules such as ZO-1, JAM-A, and occludin in the duodenum are shown in Fig 4A-C. In the SS group, PPI significantly decreased the mRNA expressions of ZO- 1 by $65 \%$, JAM-A by $45 \%$, and occludin by $58 \%$. In the WAS group, PPI significantly decreased the relative mRNA expressions of ZO-1 by $36 \%$, JAM-A by $30 \%$, and occludin by $32 \%$. The protein expressions of ZO- 1 and occludin are shown in Fig 4D, E. In the SS group, PPI significantly decreased the protein 
expressions of ZO-1 by $73 \%$ and occludin by $45 \%$ compared to those in the VT group. In the WAS group, PPI significantly decreased the protein expressions of ZO- 1 by $86 \%$ and occludin by $49 \%$ compared to those in the VT group.

\subsection{Effect of VIP receptor antagonist and mast cell stabilizer on intestinal permeability of WAS+PPI-treated mice.}

The plasma FITC concentrations in anti-VPAC (-) and anti-VPAC (+) mice under WAS+PPI-treated conditions are shown in Figure 5A. Anti-VPAC significantly decreased the plasma FITC-dx concentration by $24 \%$. The plasma FITC concentrations in KF (-) and $\mathrm{KF}(+)$ mice under WAS+PPI-treated conditions are shown in Figure 5B. KF significantly decreased the plasma FITC-dx concentration by $23 \%$. The number of duodenal mast cells in anti-VPAC $(+)$ mice under WAS+PPI-treated conditions is presented in Figure 5C. Anti-VPAC treatment significantly decreased the number of duodenal mast cells by $27 \%$. KF treatment significantly decreased the duodenal VIP levels by $44 \%$ compared to those in the WAS+PPI group (Figure 5D). These results indicated that enhancement of intestinal permeability due to WAS+PPI was mediated via VIP and activation of the mast cells. Moreover, VIP and infiltration by the mast cells functioned together to enhance intestinal permeability. 
3.10 PPI-induced exacerbation of intestinal permeability was transferable via gut

\section{microbiota transplantation}

In the WAS group, PPI-treated FMT significantly increased the intestinal permeability in vivo by $50 \%$ compared to that in vehicle-treated FMT (Figure 6A). These results showed that the gut microbiota played a role in the enhancement of intestinal permeability due to PPI under stressed condition. Vehicle-treated FMT did not affect the intestinal permeability in vivo compared to that in the PBS treatment.

3.11 PPI-treated fecal microbiota transplantation enhanced the infiltration of mast cells in the duodenum

In the WAS group, PPI-treated FMT significantly increased the number of duodenal mast cells by 1.9-fold compared to that in vehicle-treated FMT (Figure 6B). These results indicated that the gut microbiota also played a role in the enhancement of mast cell infiltration due to PPI under stressed condition.

3.12 PPI-treated fecal microbiota transplantation increased the levels of VIP in the duodenum and jejunum 
In the duodenum, PPI-treated FMT significantly increased the VIP levels by 55\% compared to those in the vehicle-treated FMT (Figure 6C). In the jejunum, PPI-treated FMT significantly increased VIP levels by $71 \%$ compared to those in the vehicle-treated FMT (Figure 6D).

\subsection{PPI changed the composition of gut microbiota}

We reduced the intestinal diversity by oral administration of large-spectrum antibiotics. After 4 weeks of large-spectrum antibiotics treatment, the composition of antibiotic feces was altered in regard to intestinal diversity compared to that of the vehicle feces. (Supplementary figure 2). The gut microbial compositions at the order level revealed that the relative abundance of Lactobacillales increased and that of Clostridiales decreased in response to PPI treatment (Figure 7A), indicating that PPI administration induced dysbiosis, which is defined as an imbalance in the composition of the intestinal microbiota. Bacterial alpha diversity was clearly different between the vehicle-treated mice and the PPI-treated mice (Figure 7B, C). A principal coordinate analysis (PCoA) for both unweighted and weighted UniFrac distance matrices revealed that the gut microbial community of PPI-treated mice was clearly different from that of the vehicle-treated mice (Figure 7D). These findings indicated that PPI administration induced an alteration in the 
Takashima et al.

microbiome in the small intestine. 


\section{DISCUSSION}

As a primary finding of this study, psychological stress induced mast cell infiltration and VIP elevation in the duodenum and jejunum, which led to increase in intestinal permeability. Besides the findings, as a new finding, we found that PPI significantly enhanced the stress-induced increase in intestinal permeability via dysbiosis of gut microbiota with increased mast cell infiltration and VIP elevation. To our knowledge, this is the first report to clarify that PPIs have additional effects on stress-induced intestinal permeability enhancement. The scheme of our findings is shown in Figure 8A.

Gut barrier function is controlled by several pathophysiological mechanisms and factors. First, CRH-mast cell-VIP axis is a predominant mechanism. Previous reports have shown that psychological stress generally increases intestinal permeability via $\mathrm{CRH}$ induction regardless of the type of stress such as WAS, communication-box-induced stress, and restraint stress. ${ }^{26}$ VIP is a neuropeptide found in mast cells and has functions to control intestinal permeability and regulate the secretion of ions and fluids from epithelial layer. Previous reports have shown that mast cells and VIP regulate both the ileal barrier function in stressed rats and colonic barrier function in patients with irritable bowel syndrome (IBS). ${ }^{6,27}$ We found that PPI-induced increase in intestinal permeability was also mediated by mast cell-VIP axis under stressed condition in the duodenum and 
jejunum, which was consistent with the previous reports; although, the type of organs involved was different. Even in the PPI treated mice, this axis may be a basic mechanism of stress-associated alteration of gut barrier function.

As a second factor associated with barrier function, a previous report showed that intestinal microbiota was one of the factors responsible for the enhancement of intestinal permeability. ${ }^{14}$ Several reports have shown that PPI can cause significant changes in gut microbiota composition. ${ }^{28,29}$ Moreover, previous studies conducted in vitro and in vivo have shown that the microbiota and probiotics are associated with intestinal barrier function. ${ }^{30,31}$ Nakajima et al. described that the gut microbial change was associated with decreased gut barrier function, as evidenced by reduced expression of the tight junction protein (TJP) genes and elevated endotoxin levels in the systemic circulation. Mouse ileal barrier dysfunction caused by chronic WAS was ameliorated by probiotic treatment. ${ }^{32}$ In this study, we have clearly shown that PPIs cause dysbiosis at the phylum and genus levels in mice small intestinal contents and PPI-treated FMT increased intestinal permeability under stressed condition, suggesting that PPI-induced dysbiosis plays a role in the enhancement of intestinal permeability under stressed condition.

As a third factor associated with disrupted mucosal barrier integrity, the alteration of TJPs is possible. Apical junctional complex including TJPs is an important factor in 
paracellular transport. TJPs are composed of many kinds of proteins such as occludin, ZO-1, and claudin families. The expression profiles of TJPs affect mucosal barrier integrity; however, the detailed analysis of their profiles is very complex. There have been several reports of changes in the expression and redistribution of TJPs by pathogens and probiotics. ${ }^{33,34}$ Since PPIs inhibit the $\mathrm{H}^{+}, \mathrm{K}^{+}$-ATPase, they affect the intracellular potassium concentration and calcium homeostasis. These changes can affect the cytoskeleton, which may lead to the alteration of TJPs. ${ }^{15}$ Our results showed that VPZ had effects similar to those of PPIs, which showed that inhibiting proton pump and gastric acid secretion may be a key mechanism associated with increase in intestinal permeability. Moreover, Neunlist et al. reported that VIP plays a regulatory role in the expression of TJPs such as ZO-1. ${ }^{7}$ In the present study, PPI treatment significantly reduced protein expressions of ZO-1 and occludin in the duodenal mucosa, which may be partly responsible for increase in intestinal permeability. The cause of these alterations may be multifactorial. Several factors may be involved such as gut microbiome, inhibitory effects of the $\mathrm{H}^{+}, \mathrm{K}^{+}$-ATPase, and VIP.

The scheme of possible mechanisms of increase in intestinal permeability due to PPI is shown in Figure 8B. Interestingly, our results showed that PPI did not affect intestinal permeability under non-stressed condition. These results indicated that stress condition 
was needed to show the effects of PPI. In other words, stress is the prime condition to increase intestinal permeability. Stress-induced increase in intestinal permeability may occur with bacterial translocation to the small intestinal mucosa. Bednarska et al. reported that bacterial passage through colonic epithelium was regulated by VIP and mast cells in patients with IBS. ${ }^{27}$ Under stressed conditions, PPI-induced dysbiosis may also cause passage of harmful bacterial components. This translocation may induce stronger immune response including mast cells and enhance VIP levels, which would lead to additional effect on the stress-induced increase in intestinal permeability. Based on our observation of increase in VIP levels due to PPI-treated FMT, dysbiosis of small intestinal flora caused by PPI may influence the mast cell-VIP pathway. Moreover, in future studies, it would be of interest to test the preventive effect of probiotics such as bifidobacteria on lactobacilli in this experimental setting. In clinical settings, increased intestinal permeability in the duodenum and jejunum may enhance microinflammation and visceral hypersensitivity to ultimately aggravate abdominal pain and diarrhea. Therefore, we consider that careful administration of PPI is needed for the stressful patients with functional GI disorders including FD.

In conclusion, under stressed conditions, PPI enhances intestinal permeability via dysbiosis of gut microbiota. VIP and mast cells are also implicated in the underlying 
27

Takashima et al.

mechanisms of this process. 


\section{ACKNOWLEDGEMENT}

We thank Emi Yoshioka for providing technical assistance.

\section{CONFLICT OF INTEREST}

Yasuhiro Fujiwara is currently conducting research sponsored by Daiichi Sankyo Corp., Ltd., and EA Pharma Co., Ltd. The other authors declare no conflicts of interest.

\section{AUTHOR CONTRIBUTION}

ST and FT designed the research, performed the research, and wrote the manuscript. ST, FT, YK, YU, KF, SI, and SU analyzed the data. Yuji Nadatani, KO, SH, Yasuaki Nagami, NK, KT, TT, TW, and YF contributed to critical revisions of the manuscript for important intellectual content. All authors read and approved the final manuscript. 


\section{REFERENCES}

1. Tominaga K, Higuchi K, Iketani T, et al. Comparison of gastrointestinal symptoms and psychological factors of functional dyspepsia to peptic ulcer or panic disorder patients. Inflammopharmacology. 2007;15(2):84-89.

2. Vanheel H, Vicario M, Vanuytsel T, et al. Impaired duodenal mucosal integrity and low-grade inflammation in functional dyspepsia. Gut. 2014;63(2):262-271.

3. Tanaka F, Tominaga K, Fujikawa Y, et al. Concentration of Glial Cell Line-Derived Neurotrophic Factor Positively Correlates with Symptoms in Functional Dyspepsia. Dig Dis Sci. 2016;61(12):3478-3485.

4. Cameron HL, Perdue MH. Stress impairs murine intestinal barrier function: improvement by glucagon-like peptide-2. J Pharmacol Exp Ther. 2005;314(1):214220.

5. Vanuytsel T, van Wanrooy S, Vanheel H, et al. Psychological stress and corticotropinreleasing hormone increase intestinal permeability in humans by a mast celldependent mechanism. Gut 2014;63(8):1293-1299.

6. Keita AV, Carlsson AH, Cigéhn M, et al. Vasoactive intestinal polypeptide regulates barrier function via mast cells in human intestinal follicle-associated epithelium and during stress in rats. Neurogastroenterol Motil. 2013;25(6):e406-417. 
7. Neunlist M, Toumi F, Oreschkova T, et al. Human ENS regulates the intestinal epithelial barrier permeability and a tight junction-associated protein ZO-1 via VIPergic pathways. Am J Physiol Gastrointest Liver Physiol. 2003;285(5):G10281036.

8. Bakker R, Dekker K, De Jonge HR, et al. VIP, serotonin, and epinephrine modulate the ion selectivity of tight junctions of goldfish intestine. Am J Physiol. 1993;264(2 Pt 2):R362-368.

9. Conlin VS, Wu X, Nguyen $\mathrm{C}$, et al. Vasoactive intestinal peptide ameliorates intestinal barrier disruption associated with Citrobacter rodentium-induced colitis. Am J Physiol Gastrointest Liver Physiol. 2009;297(4):G735-750.

10. Moayyedi P, Lacy BE, Andrews CN, et al. ACG and CAG Clinical Guideline: Management of Dyspepsia. Am J Gastroenterol. 2017;112(7):988-1013.

11. Miwa H, Kusano M, Arisawa T, et al. Evidence-based clinical practice guidelines for functional dyspepsia. J Gastroenterol. 2015;50(2):125-139.

12. Wallace JL, Syer S, Denou E, et al. Proton pump inhibitors exacerbate NSAIDinduced small intestinal injury by inducing dysbiosis. Gastroenterology. 2011;141(4):1314-1322, 1322.e1-5.

13. Bruno G, Zaccari P, Rocco G, et al. Proton pump inhibitors and dysbiosis: Current 
knowledge and aspects to be clarified. World J Gastroenterol. 2019;25(22):27062719.

14. Yoshikawa K, Kurihara C, Furuhashi H, et al. Psychological stress exacerbates NSAID-induced small bowel injury by inducing changes in intestinal microbiota and permeability via glucocorticoid receptor signaling. J Gastroenterol. 2017;52(1):6171.

15. Mullin JM, Valenzano MC, Whitby M, et al. Esomeprazole induces upper gastrointestinal tract transmucosal permeability increase. Aliment Pharmacol Ther. 2008;28(11-12):1317-1325.

16. Aguilera M, Vergara P, Martínez V. Stress and antibiotics alter luminal and walladhered microbiota and enhance the local expression of visceral sensory-related systems in mice. Neurogastroenterol Motil. 2013;25(8):e515-529.

17. Williams KL, Fuller CR, Dieleman LA, et al. Enhanced survival and mucosal repair after dextran sodium sulfate-induced colitis in transgenic mice that overexpress growth hormone. Gastroenterology. 2001;120(4):925-937.

18. Tanaka F, Tominaga K, Ochi M, et al. Exogenous administration of mesenchymal stem cells ameliorates dextran sulfate sodium-induced colitis via anti-inflammatory action in damaged tissue in rats. Life Sci. 2008;83(23-24):771-779. 
19. Gregory JC, Buffa JA, Org E, et al. Transmission of atherosclerosis susceptibility with gut microbial transplantation. J Biol Chem. 2015;290(9):5647-5660.

20. Wang Z, Klipfell E, Bennett BJ, et al. Gut flora metabolism of phosphatidylcholine promotes cardiovascular disease. Nature. 2011;472(7341):57-63.

21. Hansen AK, Krych $Ł$, Nielsen DS, et al. A Review of Applied Aspects of Dealing with Gut Microbiota Impact on Rodent Models. ILAR J. 2015;56(2):250-264.

22. Rakoff-Nahoum S, Paglino J, Eslami-Varzaneh F, et al. Recognition of commensal microflora by toll-like receptors is required for intestinal homeostasis. Cell. 2004;118(2):229-241.

23. Usui Y, Kimura Y, Satoh T, et al. Effects of long-term intake of a yogurt fermented with Lactobacillus delbrueckii subsp. bulgaricus 2038 and Streptococcus thermophilus 1131 on mice. Int Immunol. 2018;30(7):319-331.

24. Eri T, Kawahata K, Kanzaki T, et al. Intestinal microbiota link lymphopenia to murine autoimmunity via PD-1+CXCR5-/dim B-helper T cell induction. Sci Rep. 2017;7:46037.

25. Kanda Y. Investigation of the freely available easy-to-use software 'EZR' for medical statistics. Bone Marrow Transplantation. 2013;48(3):452-458.

26. Sun Y, Zhang M, Chen CC, et al. Stress-induced corticotropin-releasing hormone- 
mediated NLRP6 inflammasome inhibition and transmissible enteritis in mice. Gastroenterology. 2013;144(7):1478-1487, 1487.e1-8.

27. Bednarska O, Walter SA, Casado-Bedmar M, et al. Vasoactive Intestinal Polypeptide and Mast Cells Regulate Increased Passage of Colonic Bacteria in Patients With Irritable Bowel Syndrome. Gastroenterology. 2017;153(4):948-960.e3.

28. Tsuda A, Suda W, Morita H, et al. Influence of Proton-Pump Inhibitors on the Luminal Microbiota in the Gastrointestinal Tract. Clin Transl Gastroenterol. 2015;6:e89.

29. Freedberg DE, Toussaint NC, Chen SP, et al. Proton Pump Inhibitors Alter Specific Taxa in the Human Gastrointestinal Microbiome: A Crossover Trial. Gastroenterology. 2015;149(4):883-885.e9.

30. Nakajima M, Arimatsu K, Kato T, et al. Oral Administration of P. gingivalis Induces Dysbiosis of Gut Microbiota and Impaired Barrier Function Leading to Dissemination of Enterobacteria to the Liver. PLoS One. 2015;10(7):e0134234.

31. Kato T, Yamazaki K, Nakajima M, et al. Oral Administration of Porphyromonas gingivalis Alters the Gut Microbiome and Serum Metabolome. mSphere. 2018;3(5). pii: e00460-18.

32. Zareie M, Johnson-Henry K, Jury J, et al. Probiotics prevent bacterial translocation 
and improve intestinal barrier function in rats following chronic psychological stress.

Gut. 2006;55(11):1553-1560.

33. Wu Z, Nybom P, Magnusson KE. Distinct effects of Vibrio cholerae haemagglutinin/protease on the structure and localization of the tight junctionassociated proteins occludin and ZO-1. Cell Microbiol. 2000;2(1):11-17.

34. Karczewski J, Troost FJ, Konings I, et al. Regulation of human epithelial tight junction proteins by Lactobacillus plantarum in vivo and protective effects on the epithelial barrier. Am J Physiol Gastrointest Liver Physiol. 2010;298(6):G851-859. 


\section{FIGURE LEGENDS}

FIGURE 1 The comparison of body weight, food intake, and microscopic inflammation among the 4 groups of mice; sham stress (SS)+vehicle treatment (VT), SS+proton pump inhibitor (PPI), water avoidance stress (WAS)+VT, and WAS+PPI. (A) Daily weight change. (B) Daily food intake. Histological images of the duodenum (C) and jejunum (D). Scale bar $=100 \mu \mathrm{m}$. Disease activity index (DAI) score based on the histological assessment in the duodenum (E) and jejunum (F). $\mathrm{N}=8-10$ per group. 
Fig. 1

A

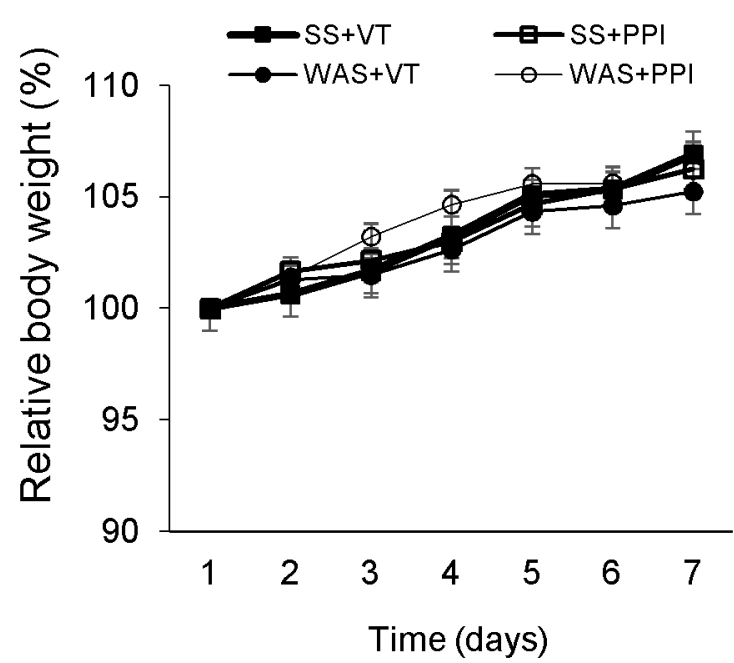

C

Duodenum

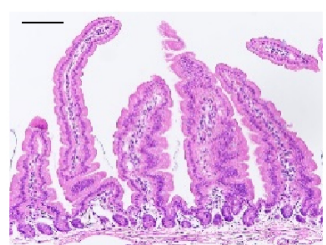

SS+VT

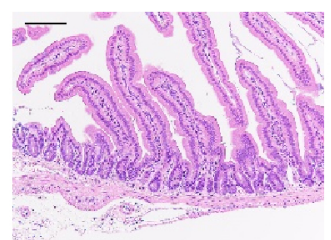

WAS+VT
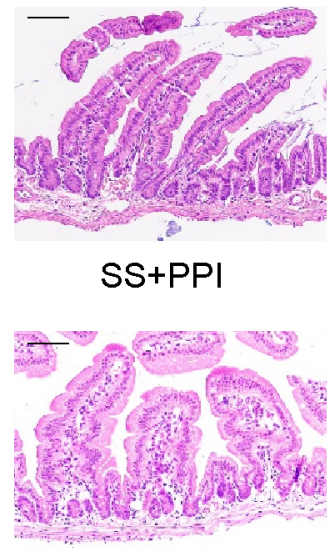

WAS+PPI

B

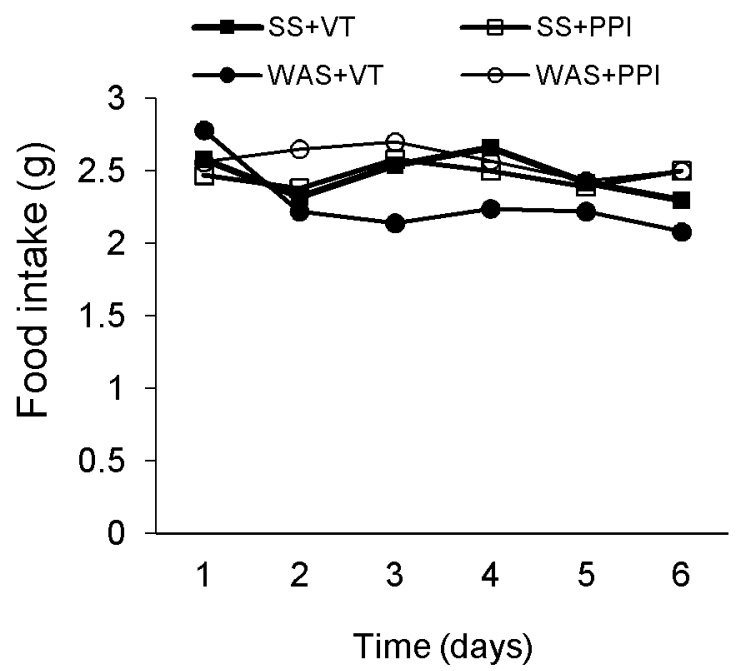

D

Jejunum

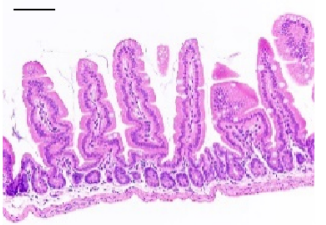

$\mathrm{SS}+\mathrm{VT}$

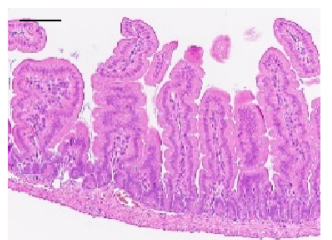

WAS+VT

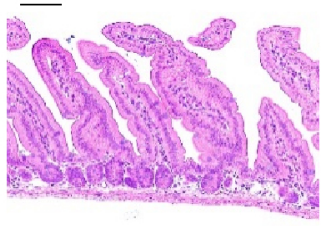

SS+PPI

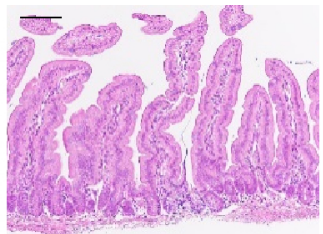

WAS+PPI
E

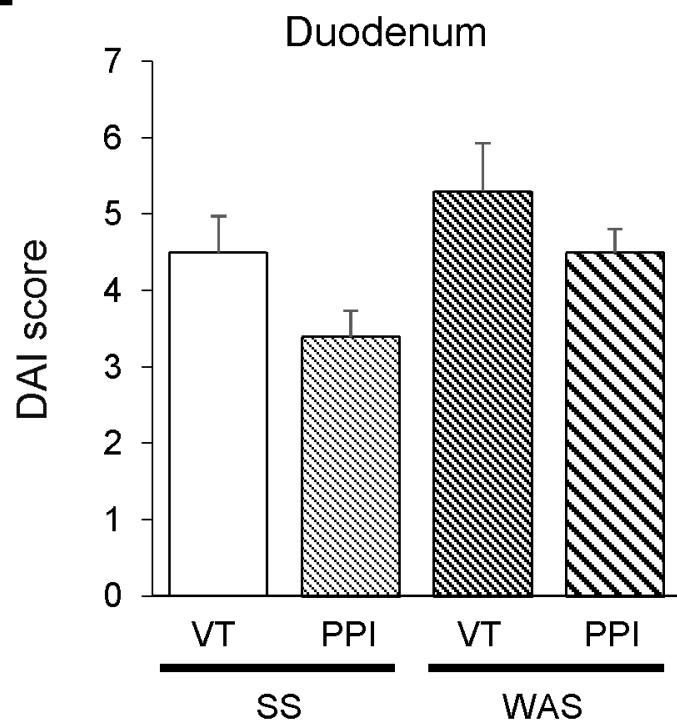

F

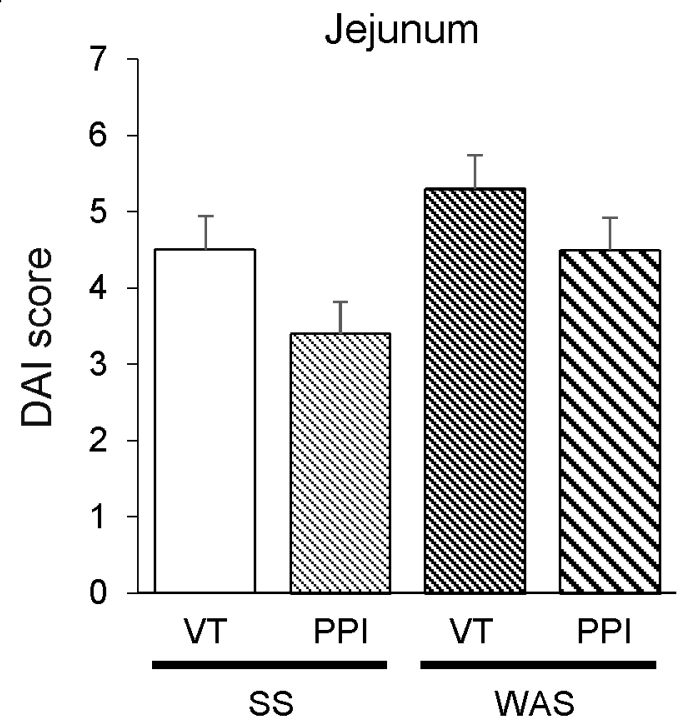


FIGURE 2 The comparison in the levels of serum corticosterone, duodenal vasoactive intestinal peptide (VIP), and duodenal mast cell counts among the 4 groups. (A) The levels of serum corticosterone. (B) The protein levels of duodenal VIP. (C) Immunofluorescence for duodenal mast cells. Scale bar $=500 \mu \mathrm{m}$. (D) Mast cell counts in the duodenum. $\mathrm{N}=8-10$ per group. $* P<0.05, * * P<0.01$. 
Fig. 2

A

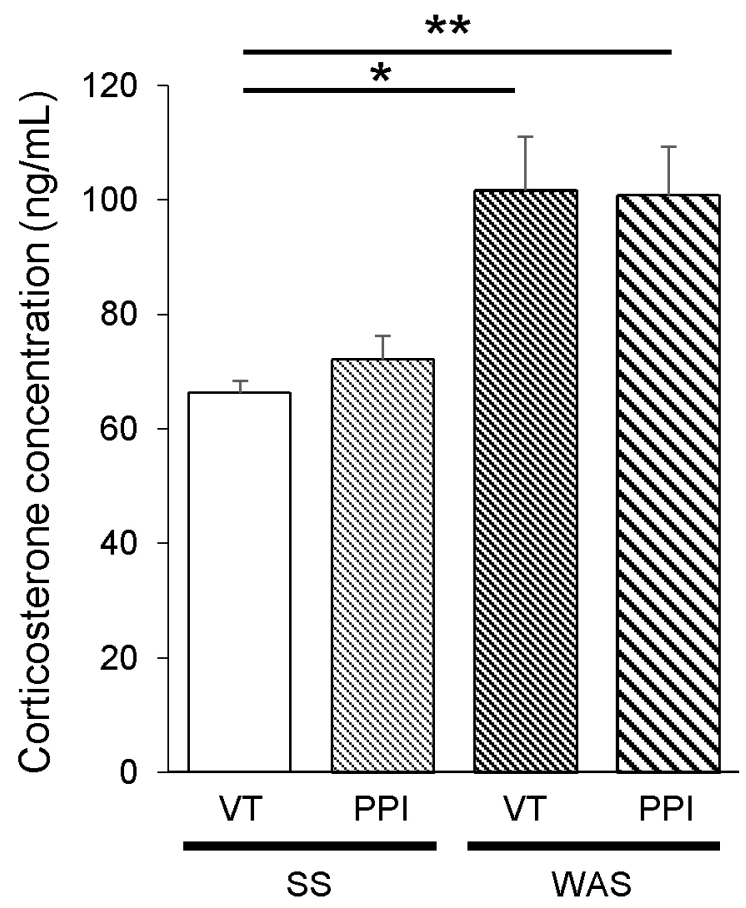

C

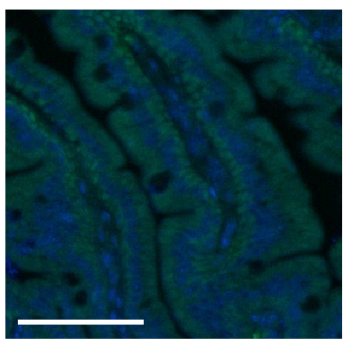

$S S+V T$

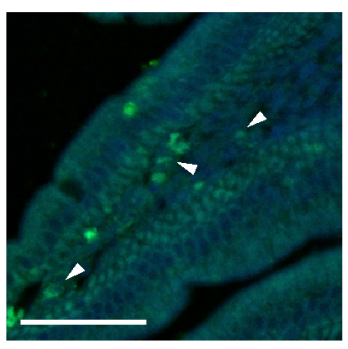

WAS+VT

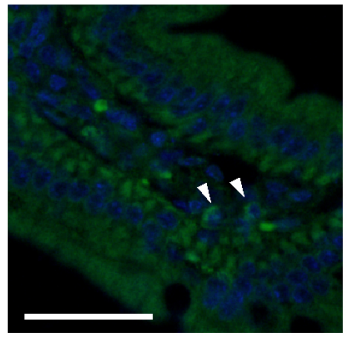

$S S+P P I$

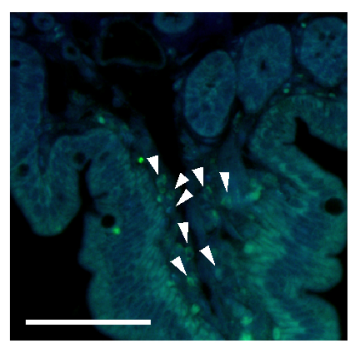

WAS+PPI
B

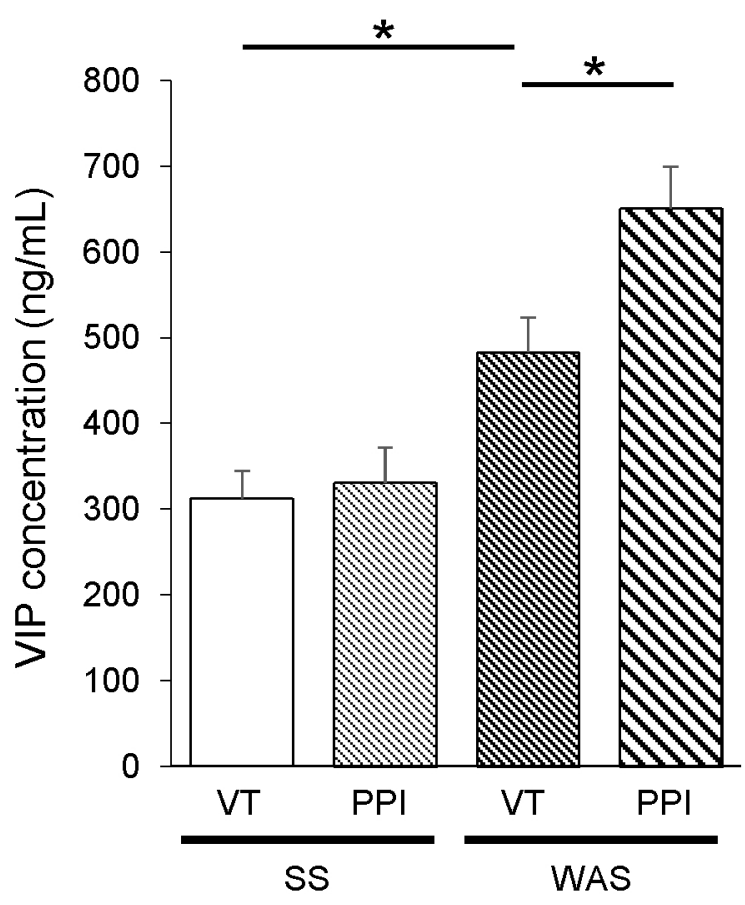

D

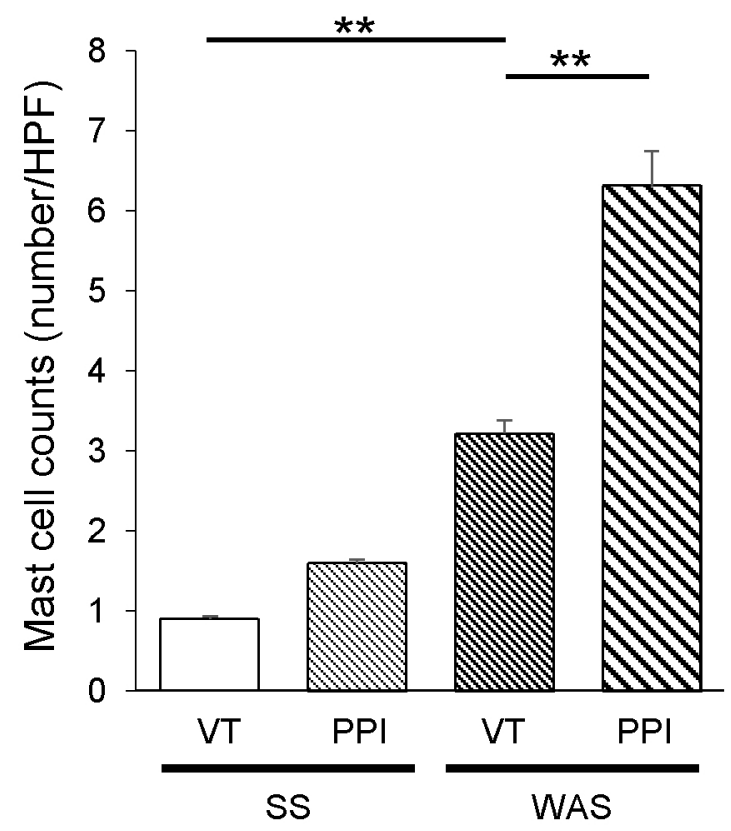


FIGURE 3 The effects of WAS, PPI, and vonoprazan (VPZ) on intestinal permeability. (A) Effect of PPI on in vivo intestinal permeability. (B) Effect of VPZ on in vivo intestinal permeability ( $\mathrm{N}=8-10$ per group). Ex vivo intestinal permeability evaluated by fluorescein isothiocyanate (FITC)-dextran flux in the duodenum (C) and jejunum (D) (N $=5-8$ per group). Ex vivo intestinal permeability evaluated by transepithelial electrical resistance (TEER) in the duodenum (E) and jejunum (F) $\left(\mathrm{N}=5-8\right.$ per each group). ${ }^{*} P<$ $0.05, * * P<0.01$ 
Fig. 3

A

B
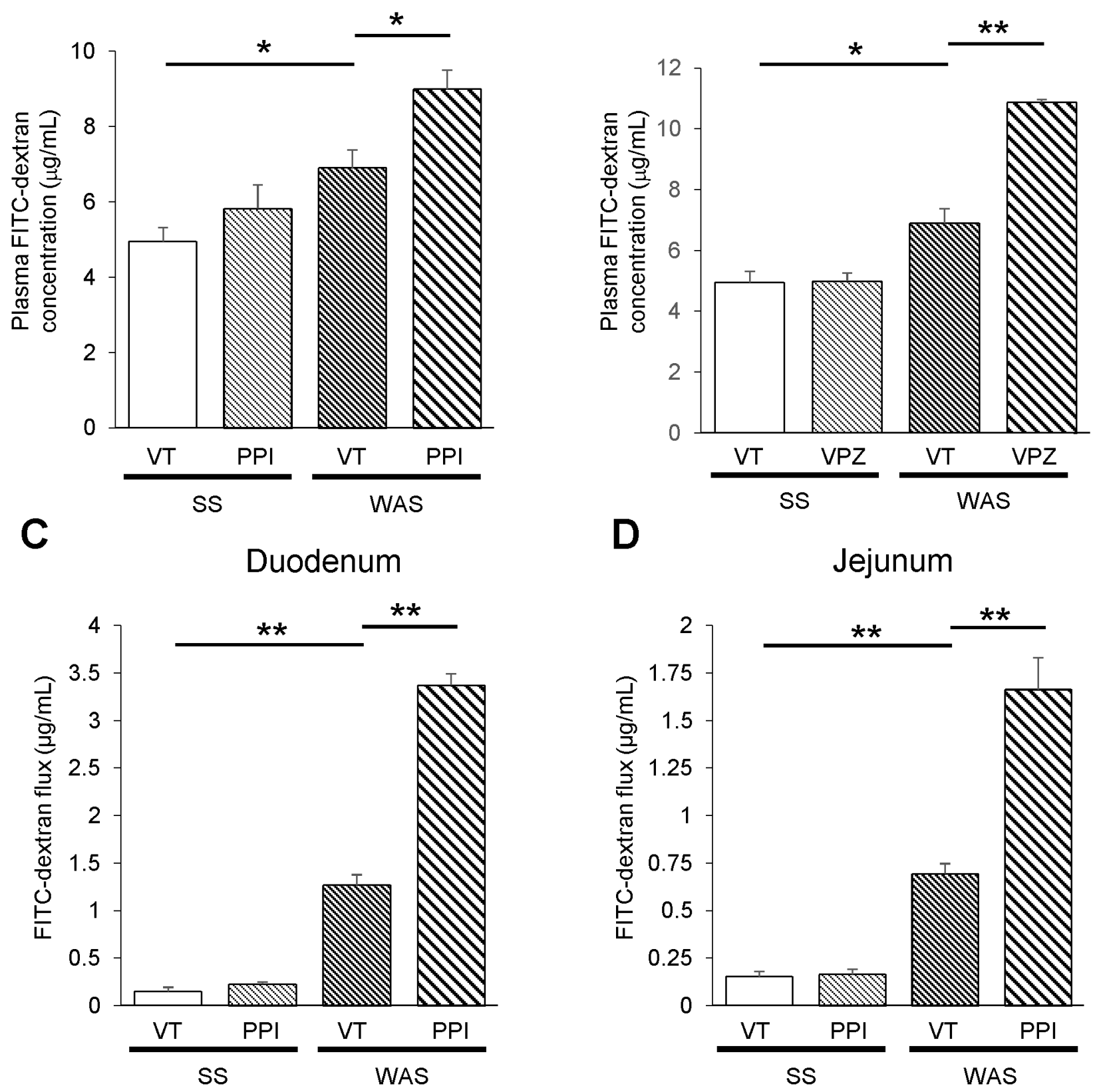

E

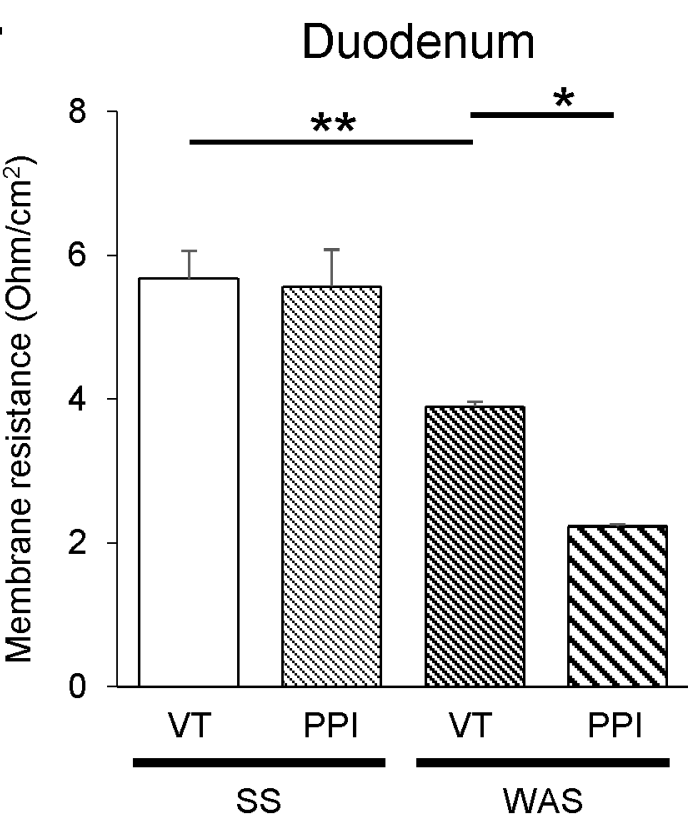

F

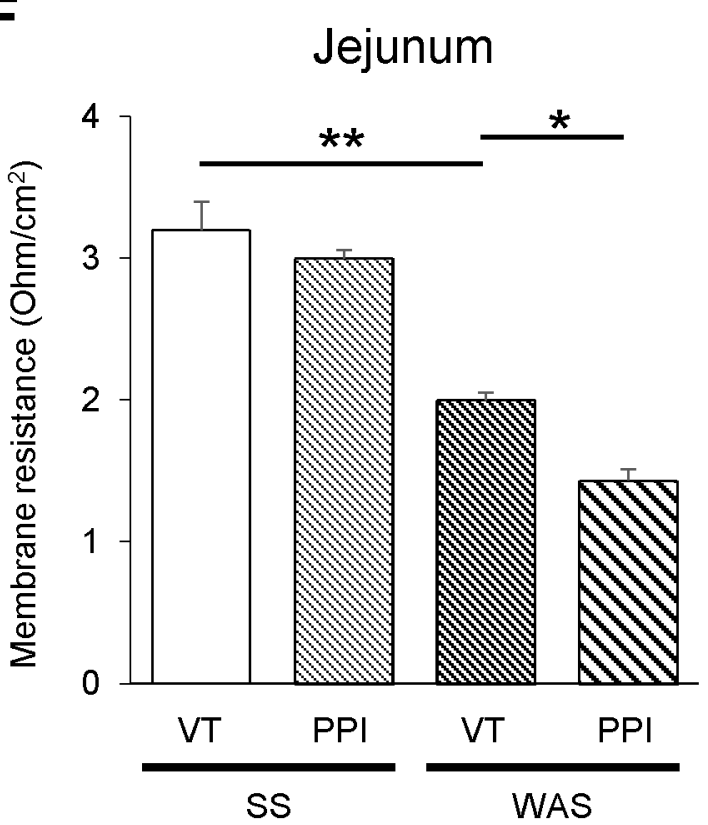


FIGURE 4 The relative mRNA expressions of cell-to-cell adhesion molecules in the duodenum: (A) Zonula occludens-1 (ZO-1), (B) Junctional adhesion molecule-A (JAM-A), and (C) Occludin. Protein expressions of (D) ZO-1 and (E) Occludin in the duodenum. N = 8-

10 per group. $* P<0.05, * * P<0.01$. 
A

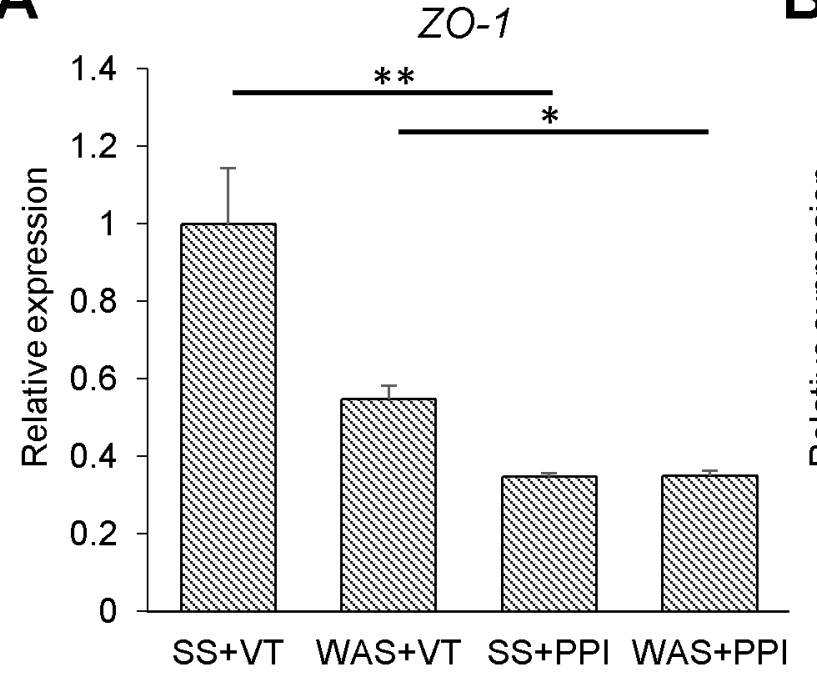

D

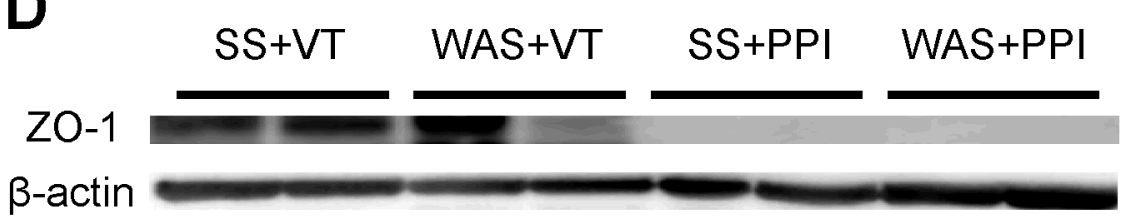

$\beta$-actin

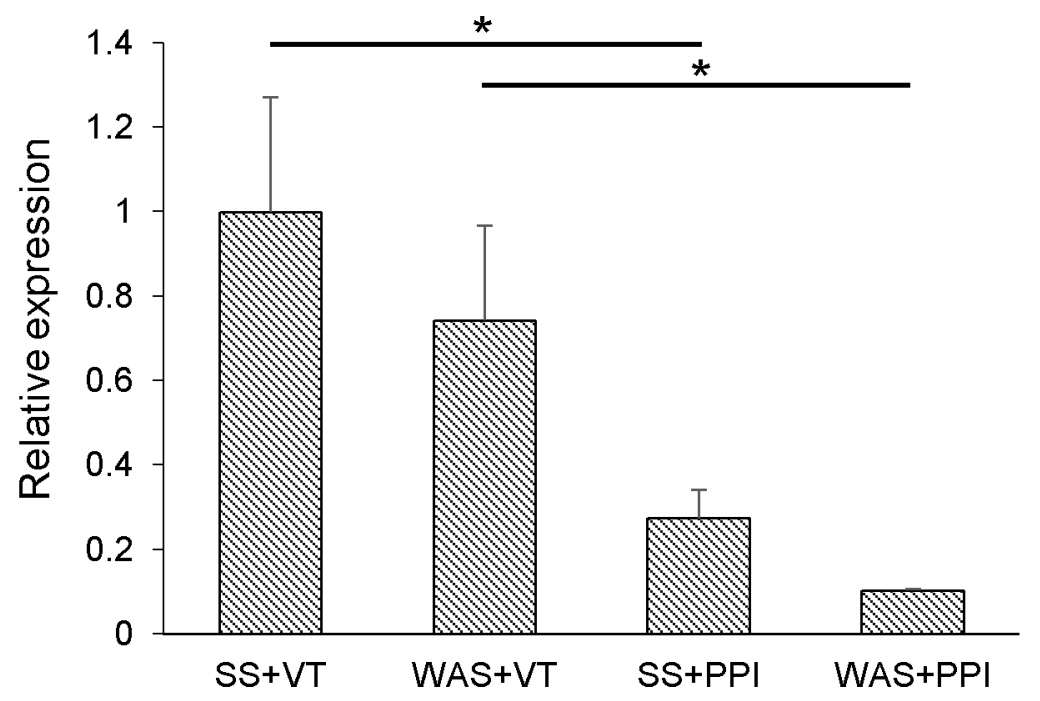

B

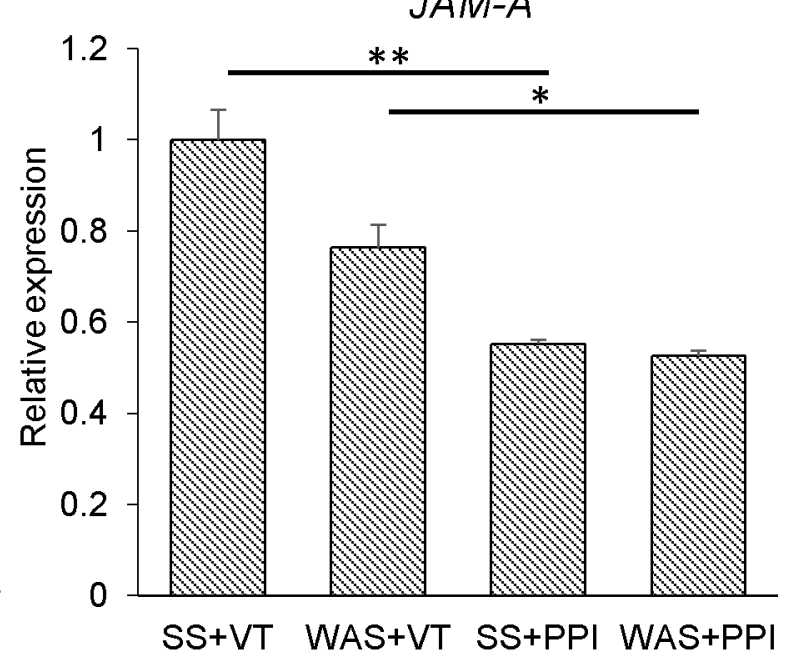

C

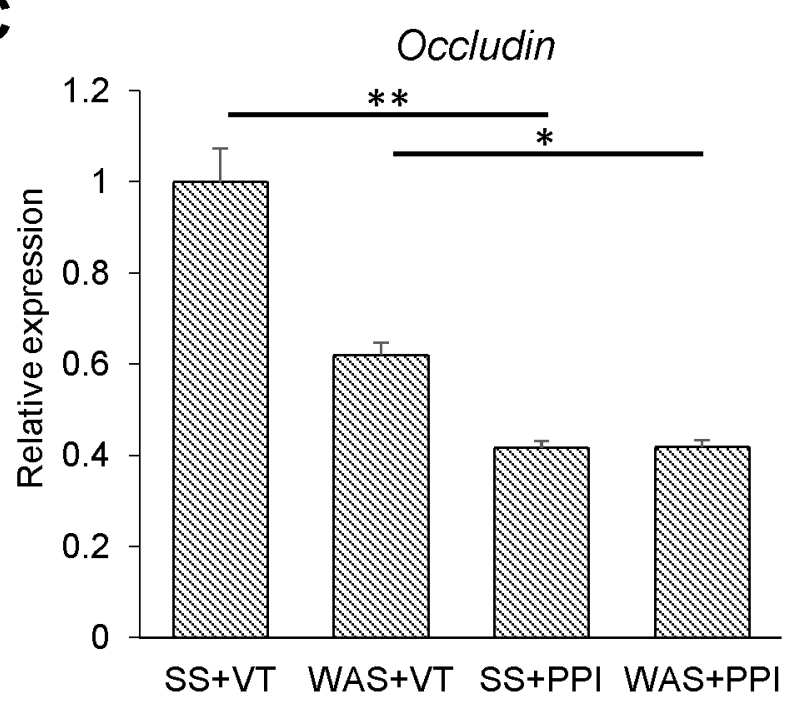

E
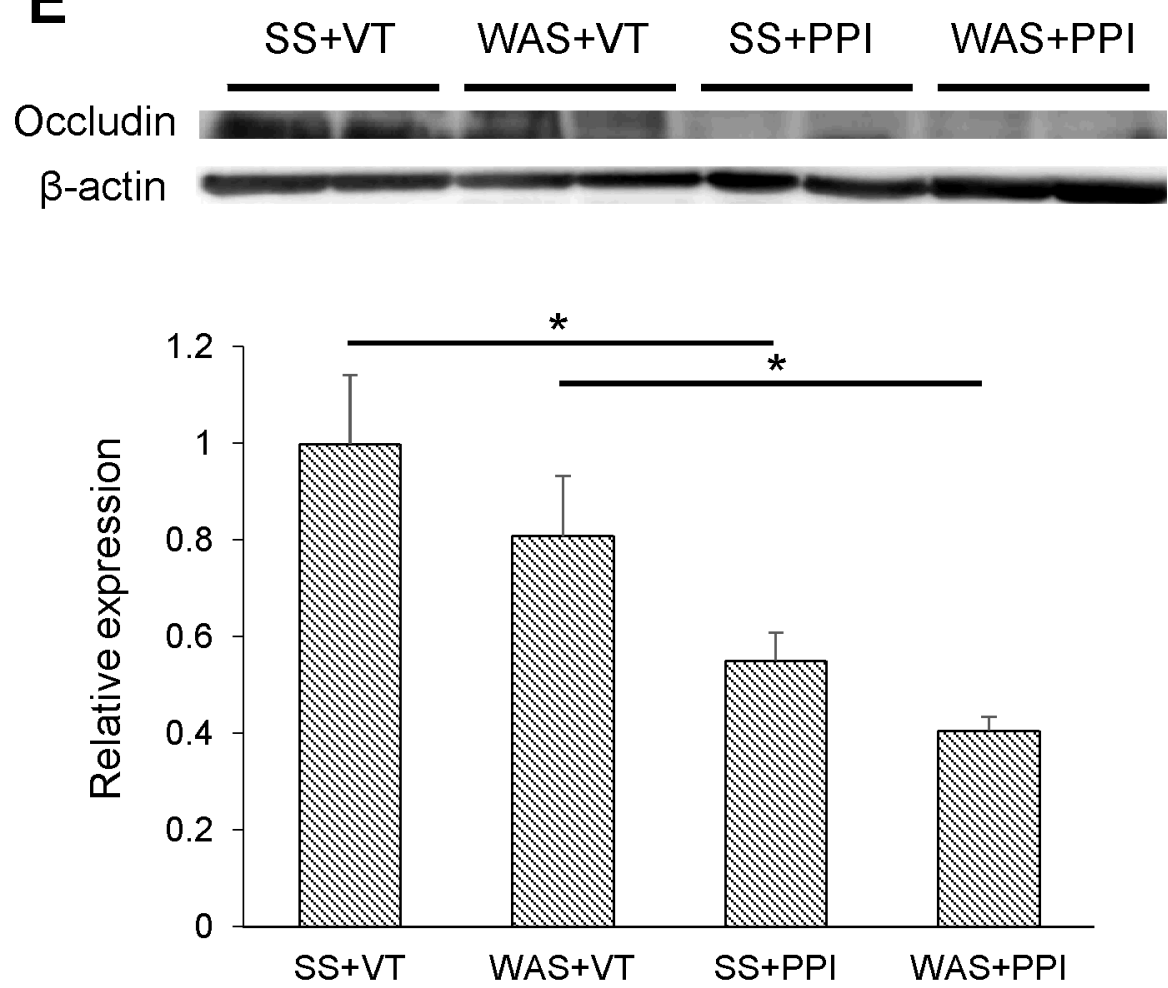
FIGURE 5 (A) Effects of vasoactive intestinal peptide receptor antagonist (anti-VPAC) on intestinal permeability in vivo in WAS+PPI-

treated mice. (B) Effects of mast cell stabilizer, ketotifen (KF), on intestinal permeability in vivo. (C) Effects of anti-VPAC treatment on mast cell counts in the duodenum. (D) Effects of KF treatment on the protein levels of duodenal VIP. N $=8-10$ per group. ${ }^{*} P<0.05, * * P<$ 0.01 . 
Fig. 5
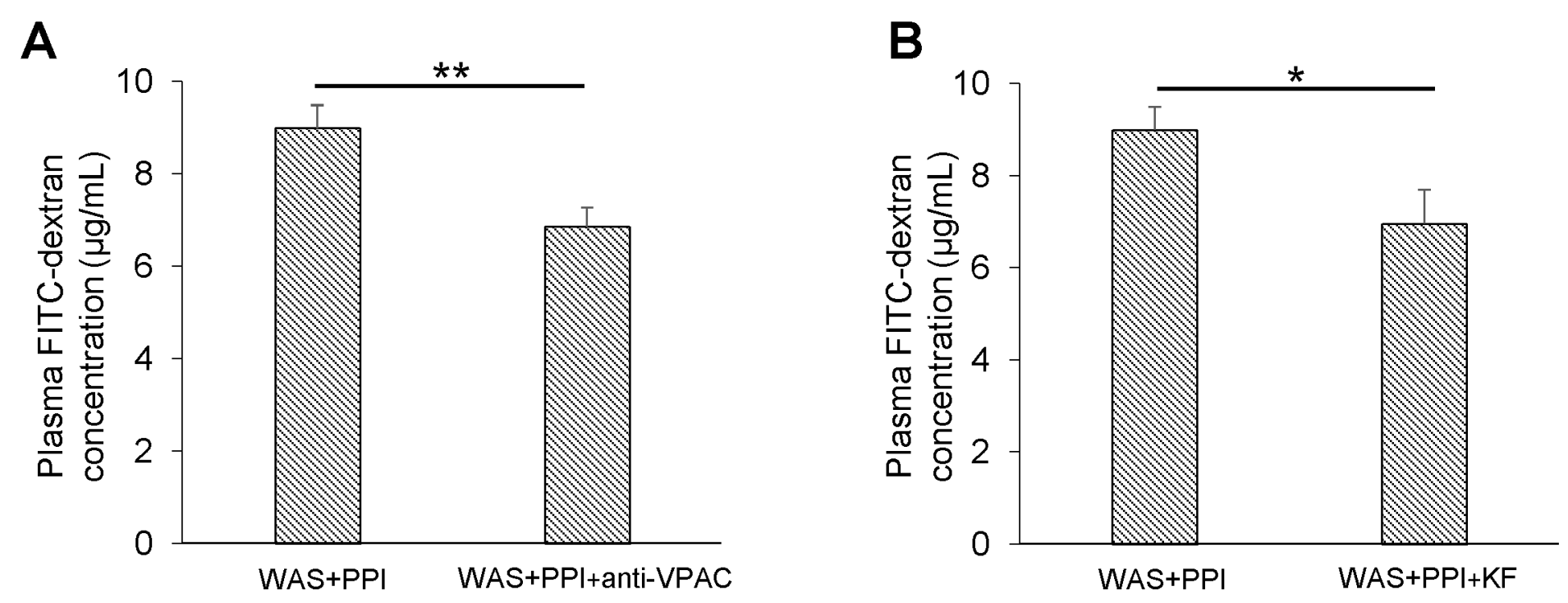

C

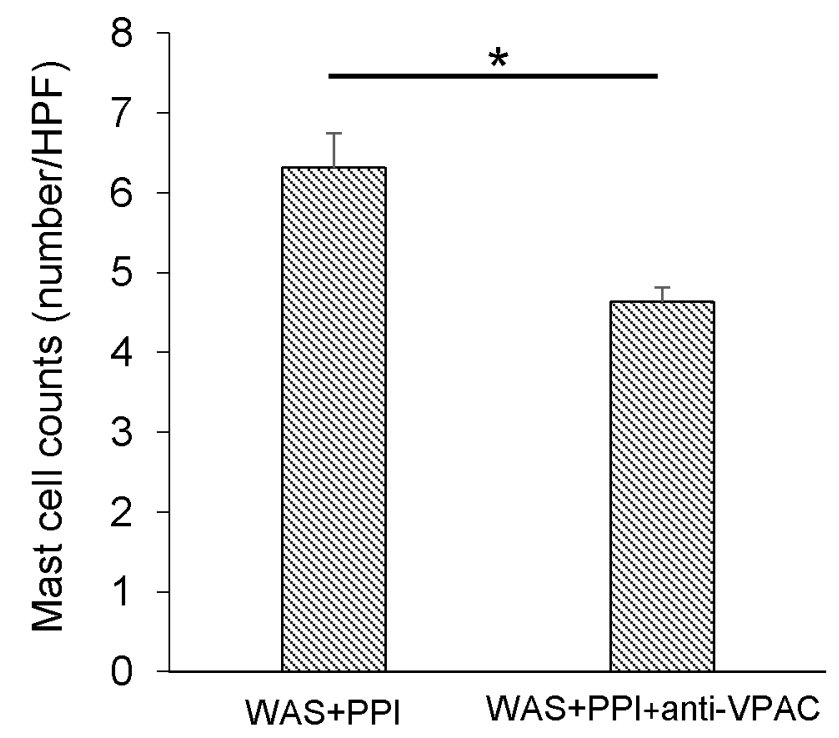

D

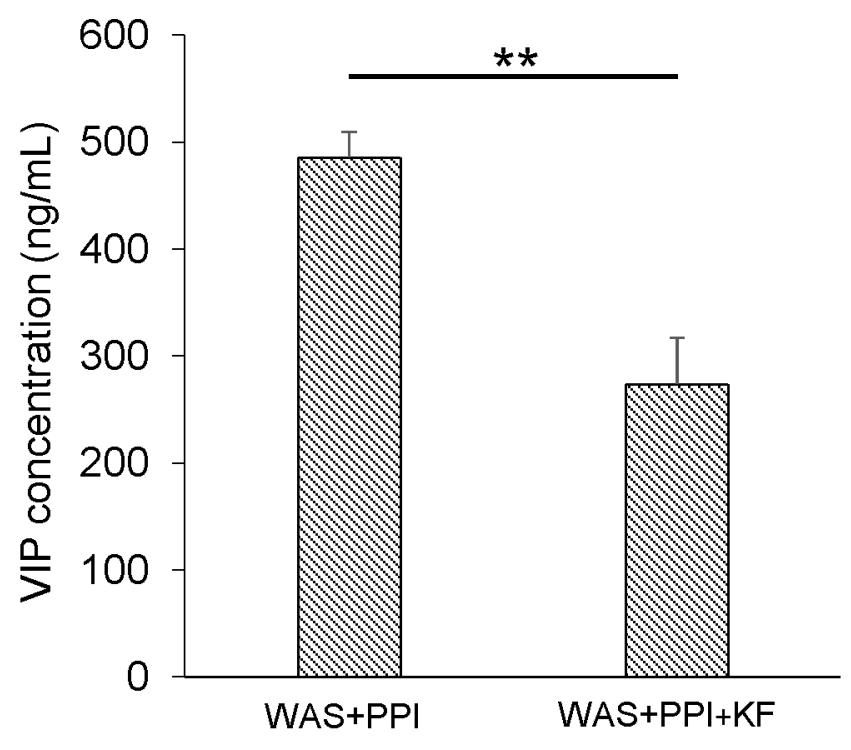


FIGURE 6 (A) In vivo intestinal permeability after phosphate-buffered saline (PBS) treatment, vehicle-treated fecal microbiota transplantation (FMT), and PPI-treated FMT ( $\mathrm{N}=5-8$ per group). (B) The comparison of mast cell counts in the duodenum between vehicle-treated and PPI-treated FMT. The comparison of levels of VIP between vehicle-treated and PPI-treated FMT in the duodenum (C) and jejunum (D) $(\mathrm{N}=5$ per group). $* P<0.05, * * P<0.01$. 
Fig. 6
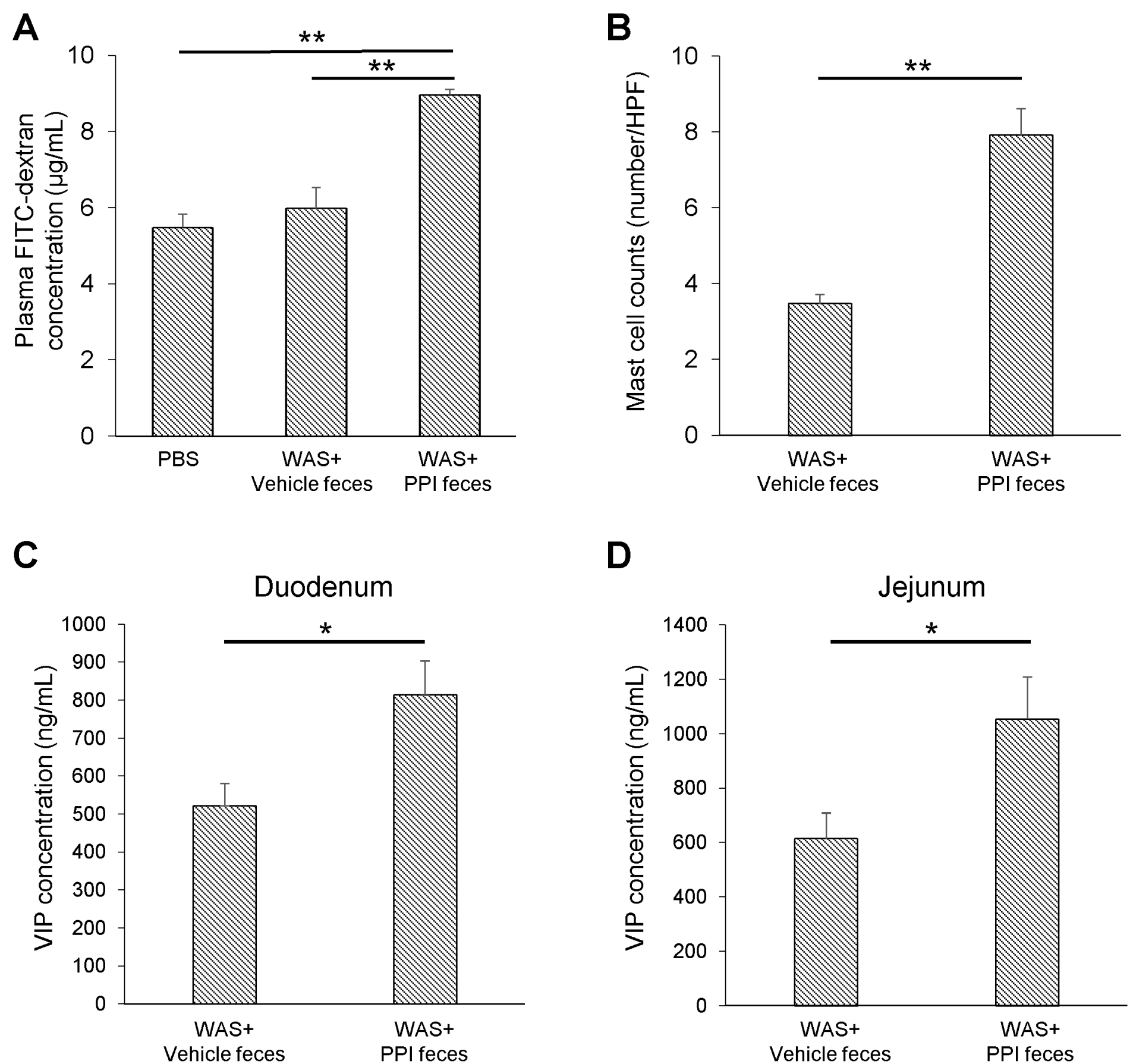
FIGURE 7 (A) The gut microbial compositions at the order level based on the relative abundance of operational taxonomic units (OTUs)

for both vehicle and PPI-treated feces. (B) Bacterial alpha diversity evaluation of vehicle and PPI-treated feces. (C) Alpha value for the factorial Kruskal-Wallis test between vehicle and PPI-treated feces. (D) A Principal coordinate analysis (PCoA) of gut microbial community according to the unweighted or weighted UniFrac distance matrices. $\mathrm{N}=7$ per group. $* P<0.05,{ }^{*} P<0.01$. 
Fig. 7

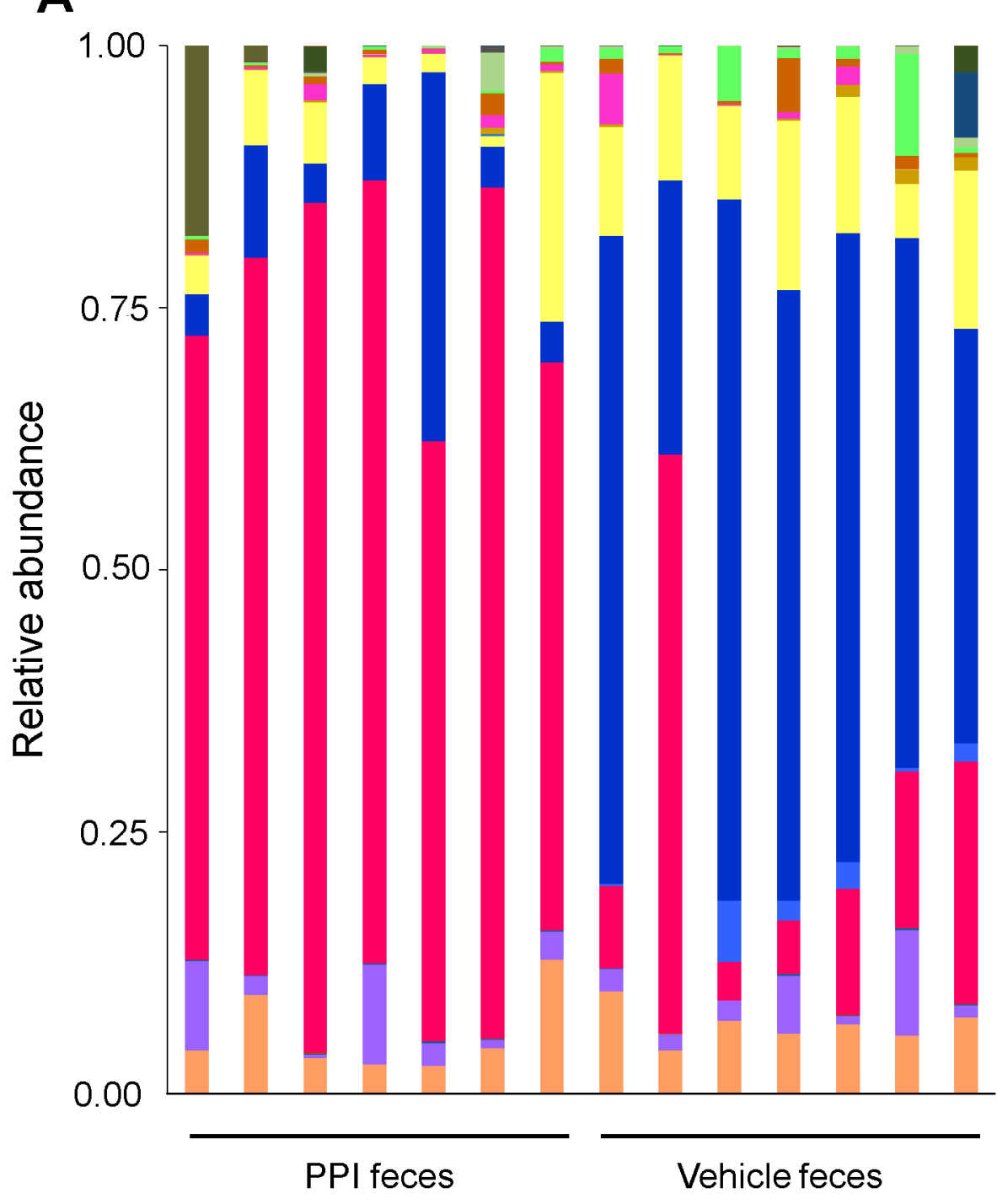

Bacterial order

- Verrucomicrobiales - Clostridiales

Desulfovibrionales Turicibacterales

- Burkholderiales $\quad$ Lactobacillales

Rickettsiales

Bacteroidales

Erysipelotrichales

Coriobacteriales
B

Alpha diversity rarefaction curve (Observed out)

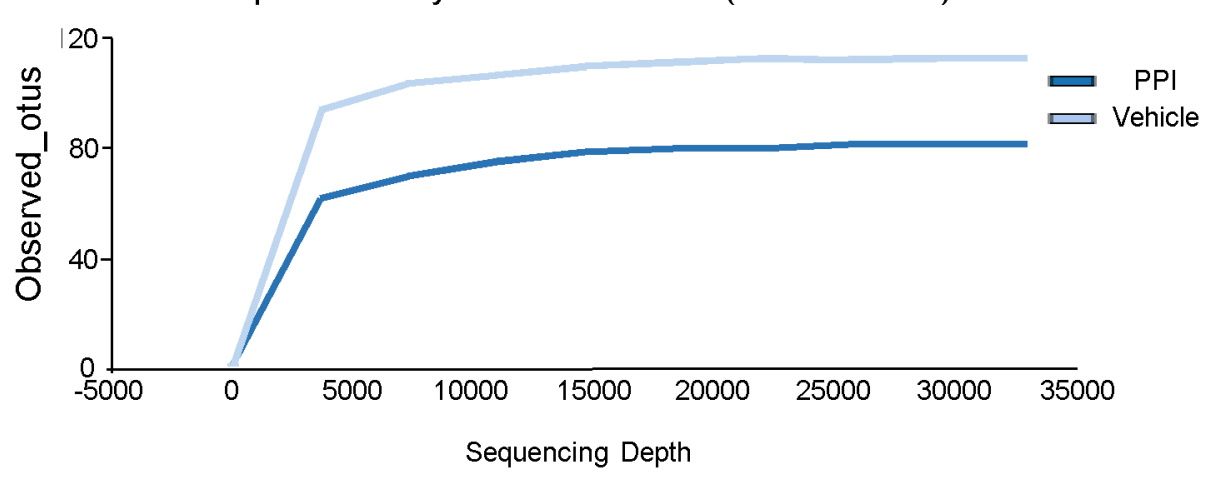

$$
\begin{aligned}
& \text { C } \\
& \text { D }
\end{aligned}
$$
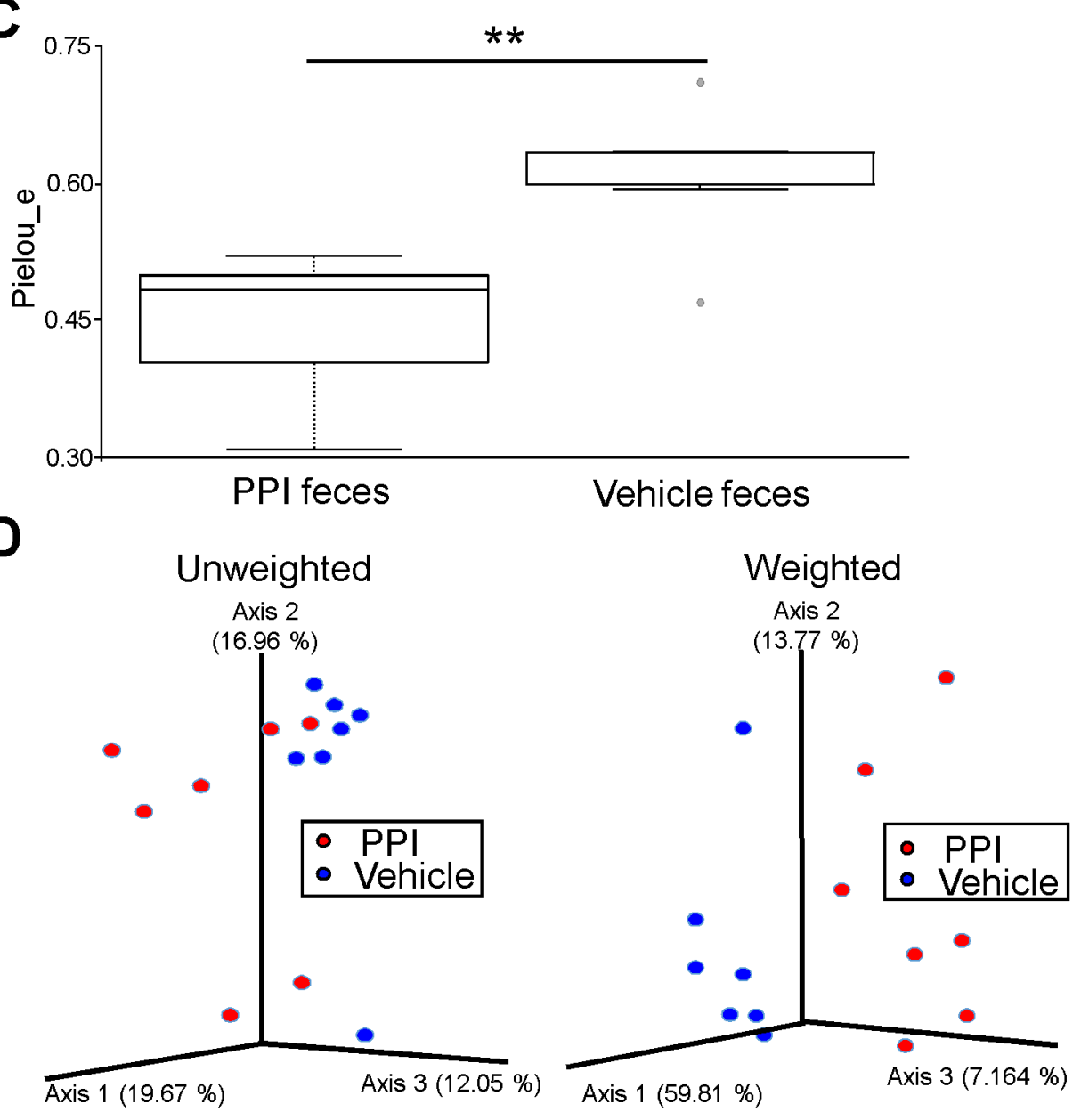
FIGURE 8 (A) The scheme of our findings. Stress induced mast cell infiltration and VIP elevation, which led to increased intestinal permeability in the duodenum and jejunum. It is also suggested that dysbiosis of small intestinal flora caused by PPI may influence this pathway. (B) The scheme of possible mechanisms of increase in intestinal permeability due to PPI. Stress-induced increase in intestinal permeability may cause bacterial translocation to the duodenal and jejunal mucosa. PPI-induced dysbiosis may also cause passage of harmful bacterial components. This translocation may induce stronger immune response including mast cells and enhance VIP levels, which may lead to additional effects on increased intestinal permeability. 
A

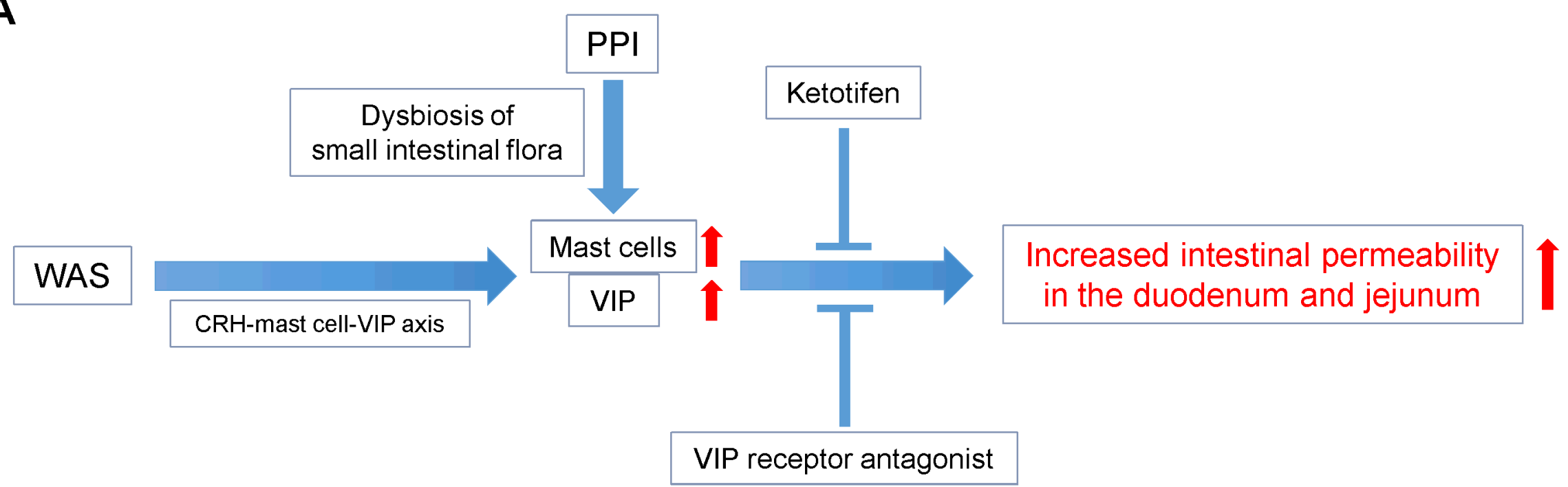

B

\section{Stressed condition}

Intestinal permeability

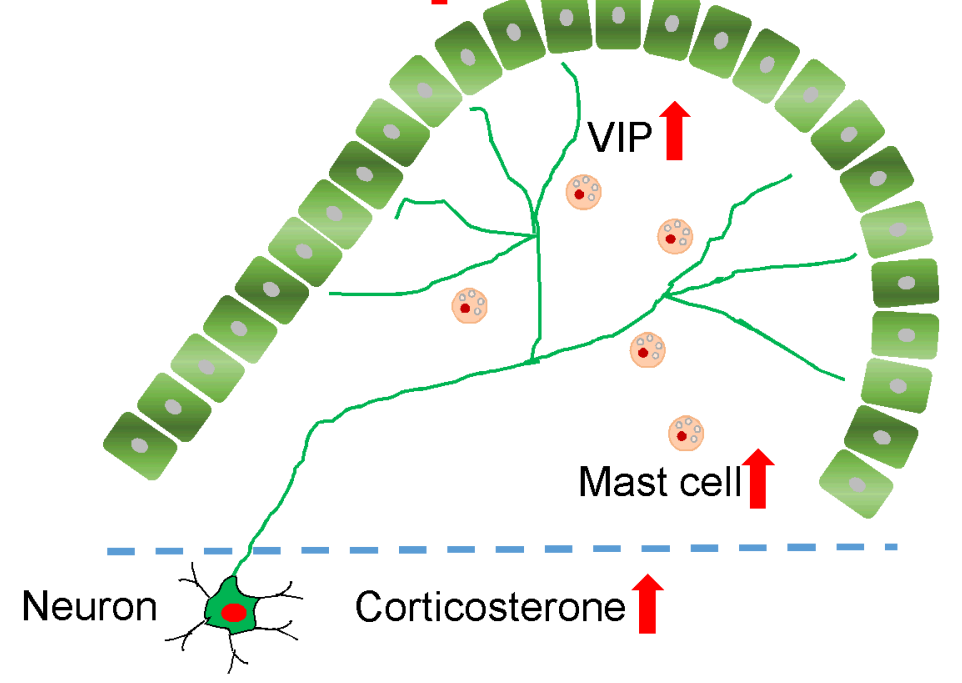

\section{Dysbiosis}

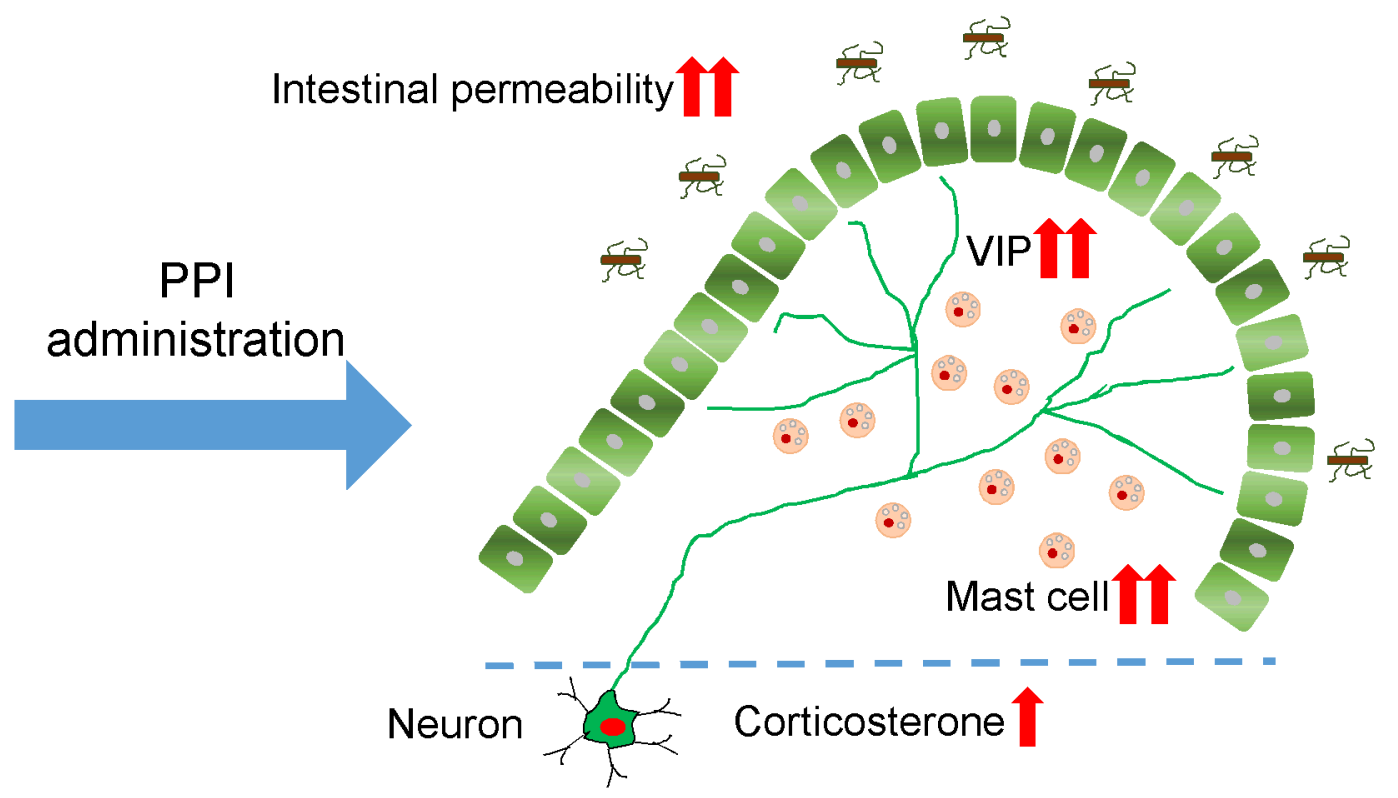


SUPPLEMENTARY FIGURE 1 The effects of WAS, omeprazole (OPZ), and lansoprazole (LPZ) treatments on intestinal permeability.

(A) Effect of OPZ on in vivo intestinal permeability. (B) Effect of LPZ on in vivo intestinal permeability ( $\mathrm{N}=8$-10 per group). 
A

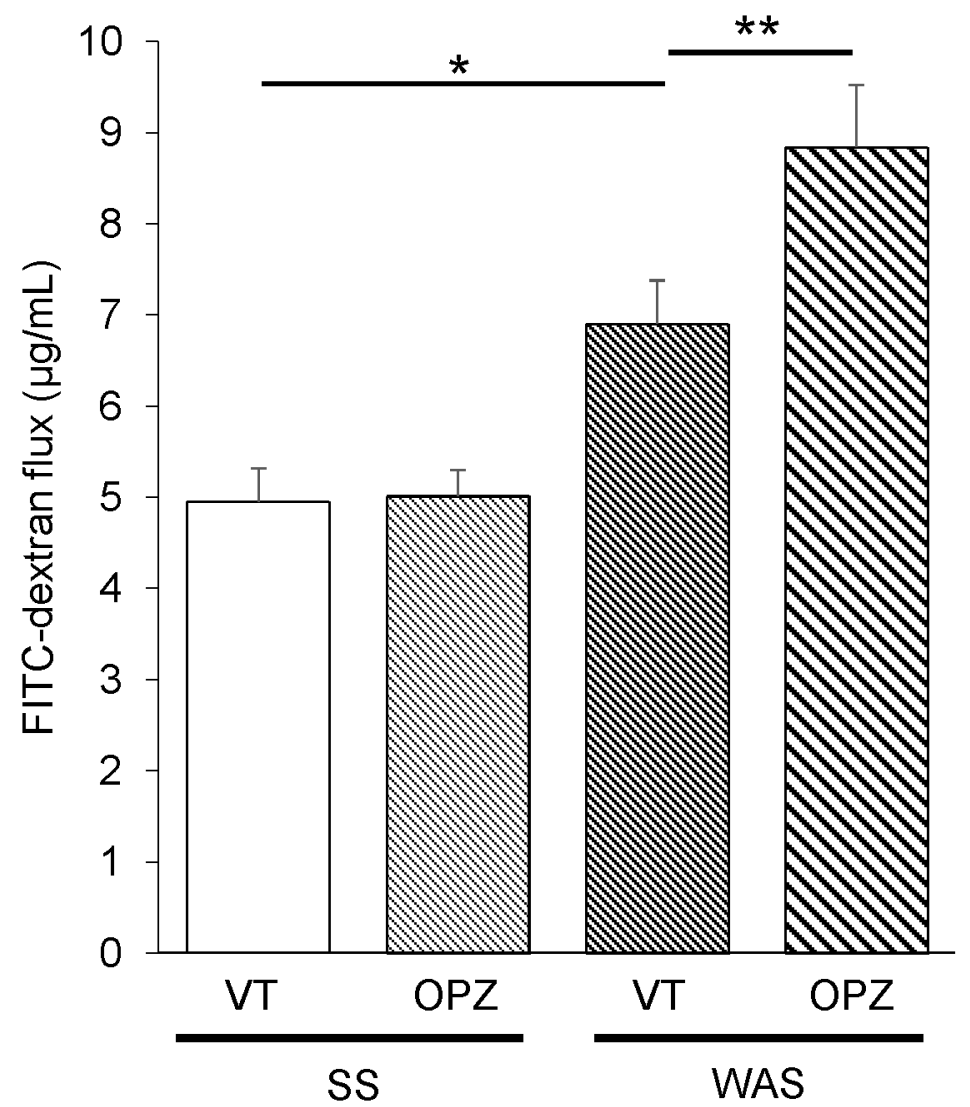

B

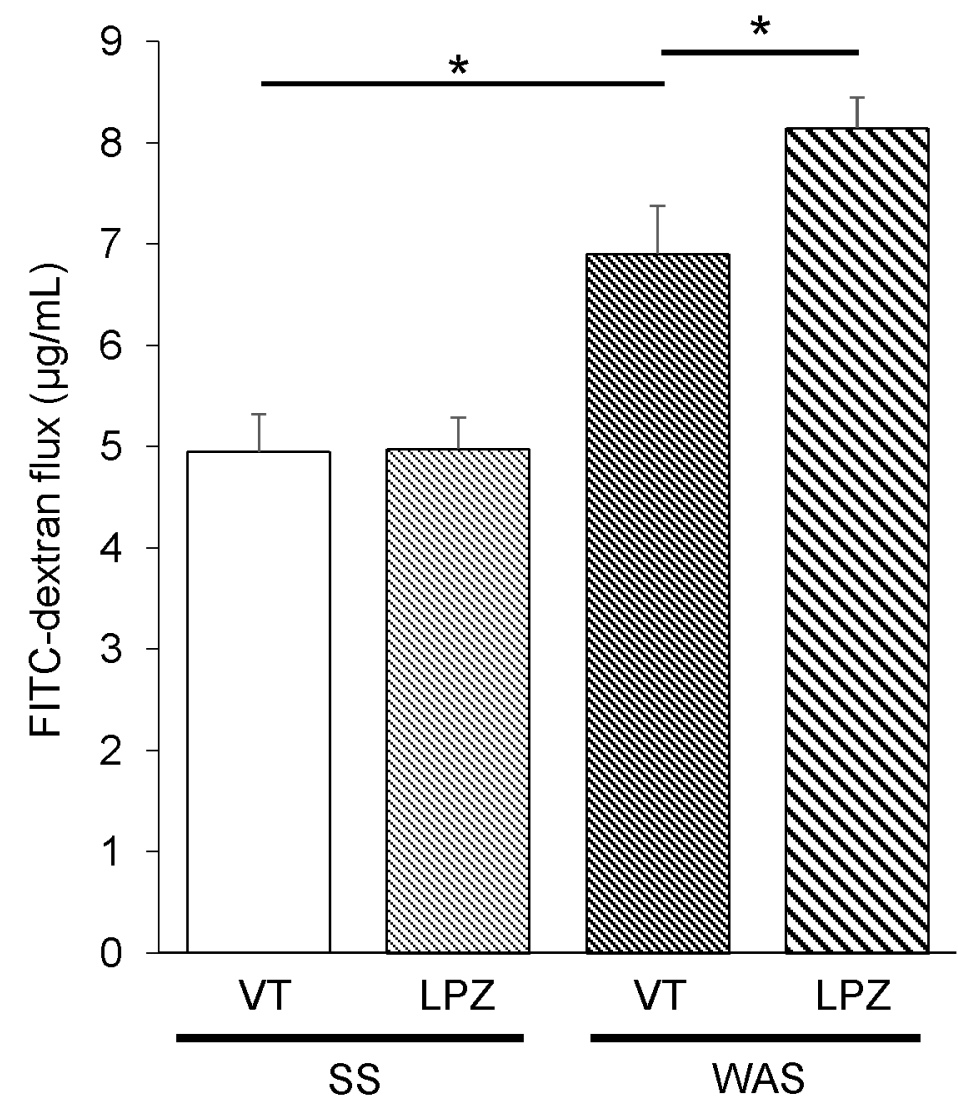


SUPPLEMENTARY FIGURE 2 (A) The gut microbial compositions at the order level based on the relative abundance of operational taxonomic units (OTUs) for both vehicle and antibiotics (ABX)-treated feces. The relative abundance of Lactobacillales and Enterobacteriales increased and that of Clostridiales and Erysipelotrichales decreased with ABX treatment. (B) Bacterial alpha diversity evaluation of vehicle and ABX-treated feces. Bacterial alpha diversity was clearly different between the vehicle-treated mice and ABXtreated mice (C) Alpha value for the factorial Kruskal-Wallis test between vehicle and ABX-treated feces. The alpha values were significantly different between the vehicle-treated mice and ABX-treated mice. (D) A Principal coordinate analysis (PCoA) of the gut microbial community according to the unweighted or weighted UniFrac distance matrices. The gut microbial community of ABX-treated mice was clearly different from that of the vehicle-treated mice in PCoA for both unweighted and weighted Unifrac distance matrices. $\mathrm{N}$

$=7$ per group. $* P<0.05, * * P<0.01$. 
Supplementary figure 2

A

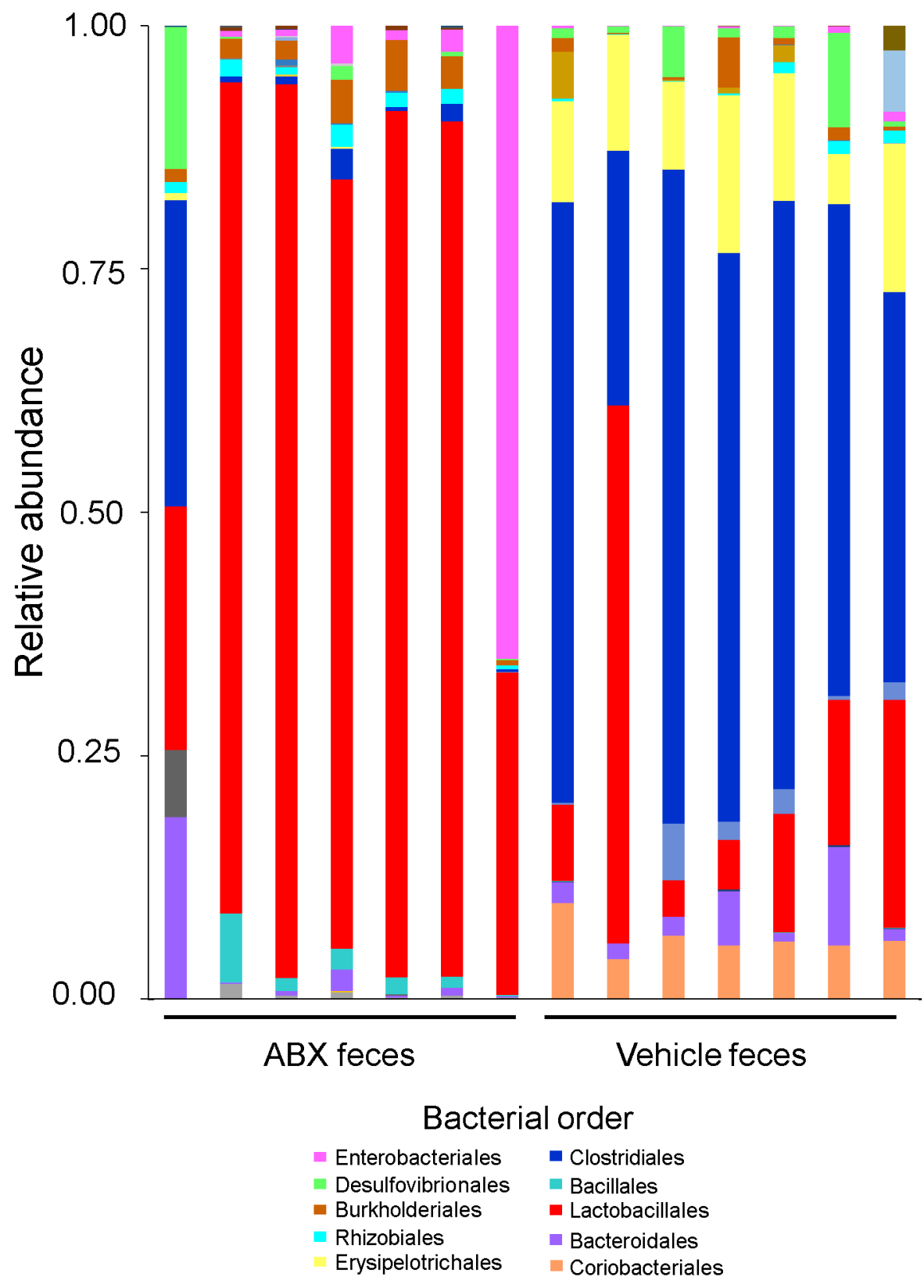

B
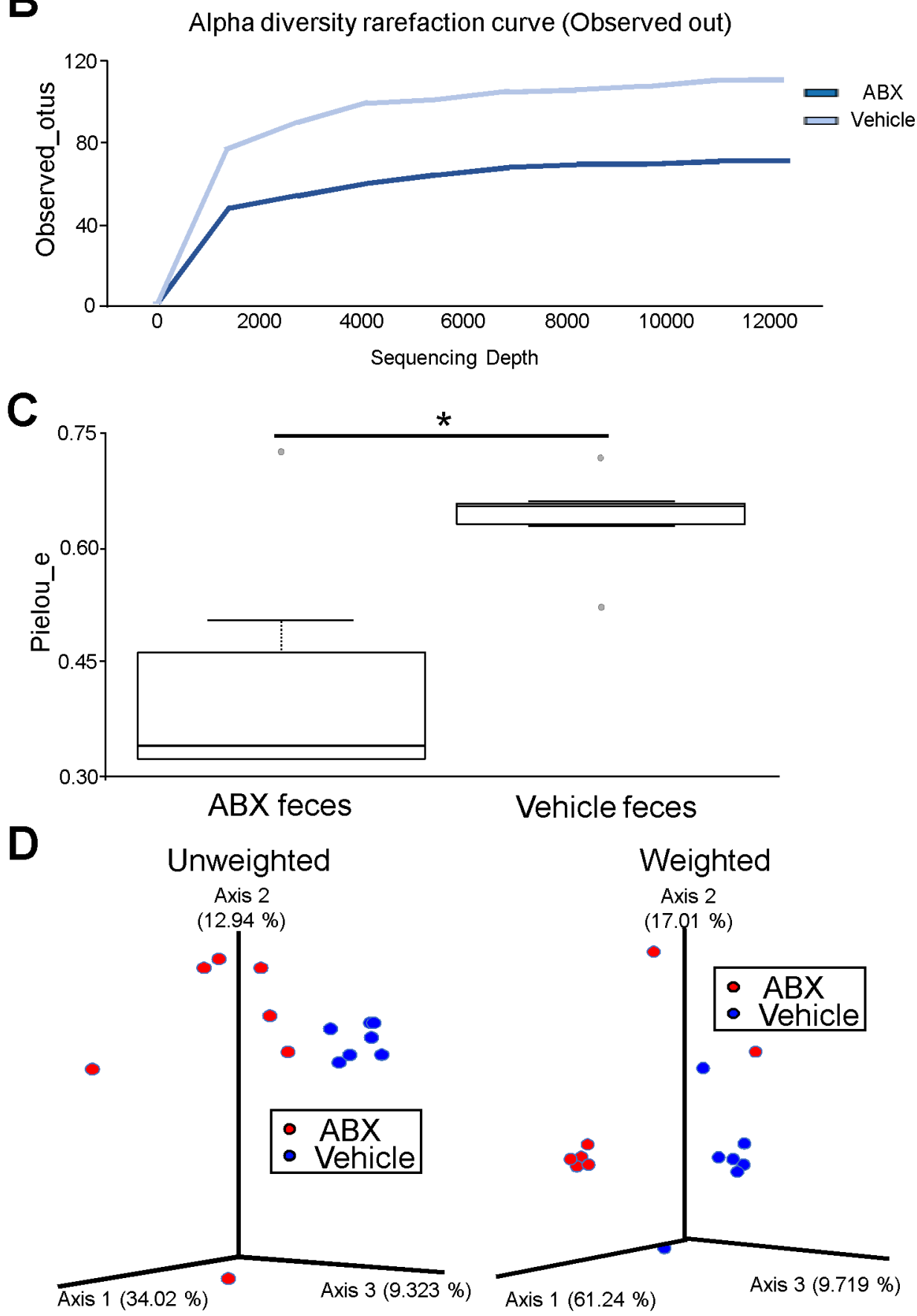